\title{
Dynamic causal modelling of induced responses
}

\author{
C.C. Chen, ${ }^{*}$ S.J. Kiebel, and K.J. Friston \\ Wellcome Trust Centre for Neuroimaging, University College London, UK
}

Received 4 October 2007; revised 17 February 2008; accepted 12 March 2008

Available online 28 March 2008

\begin{abstract}
This paper describes a dynamic causal model (DCM) for induced or spectral responses as measured with the electroencephalogram (EEG) or the magnetoencephalogram (MEG). We model the time-varying power, over a range of frequencies, as the response of a distributed system of coupled electromagnetic sources to a spectral perturbation. The model parameters encode the frequency response to exogenous input and coupling among sources and different frequencies. The Bayesian inversion of this model, given data enables inferences about the parameters of a particular model and allows us to compare different models, or hypotheses. One key aspect of the model is that it differentiates between linear and non-linear coupling; which correspond to within and betweenfrequency coupling respectively. To establish the face validity of our approach, we generate synthetic data and test the identifiability of various parameters to ensure they can be estimated accurately, under different levels of noise. We then apply our model to EEG data from a faceperception experiment, to ask whether there is evidence for non-linear coupling between early visual cortex and fusiform areas.

(c) 2008 Elsevier Inc. All rights reserved.
\end{abstract}

Keywords: Non-linear coupling; Neuronal dynamics; Induced responses; Dynamic causal modelling; Spectral; Synchronisation

\section{Introduction}

The aim of this paper is to describe a dynamic causal model for induced or spectral responses measured with the electroencephalogram (EEG) or the magnetoencephalogram (MEG). Induced oscillations are a hallmark of many neuronal systems (Gray et al., 1989; Tiitinen et al., 1993; Tass et al., 1998; Singer, 1999; Tallon-Baudry and Bertrand, 1999; Varela et al., 2001; Singh et al., 2002; TallonBaudry et al., 1997; Pantev, 1995). They are ubiquitous in both the sensory and motor systems and may play an important role in the

\footnotetext{
* Corresponding author. Wellcome Trust Centre for Neuroimaging, Institute of Neurology, UCL, 12 Queen Square, London WC1N 3BG, UK. Fax: +44 2078131445.

E-mail address: c.chen@fil.ion.ucl.ac.uk (C.C. Chen).

Available online on ScienceDirect (www.sciencedirect.com).
}

functional integration of distributed brain systems. Induced responses represent a stimulus- or task-related increase in frequency-specific power recorded electrophysiologically (Kilner et al., 2005). It had been shown that the modulation of frequencyspecific dynamics, reflecting functional integration within or between neuronal sources, can be used to characterise neuronal coupling and address questions about the underlying mechanisms in health and disease; for example, task-related changes (Kilner et al., 2002, 2004; Leocani et al., 1997), learning-related changes (Gerloff and Andres, 2002) and functional deficits (Czigler et al., 2008; Patino et al., 2006; Raethjen et al., 2007). The machinery presented in this paper contributes to this endeavour by allowing one to make inferences and quantify changes in either linear or non-linear coupling, induced experientially or associated with pathophysiology. We will demonstrate this in a future paper that asks whether backward connections exert greater non-linear influences than forward connections in visual processing hierarchies. These sorts of questions are central to understanding the nature of neuronal computations and how they are implemented in the brain. On the clinical side, the methods reported here are currently being applied to assess coupling in the motor system, during recovery from stroke.

The purpose of this work was to establish a phenomenological model of how induced responses are caused, and how they evolve dynamically, in a distributed system of coupled electromagnetic sources. Inversion of this model, given empirical data, furnishes inferences about different models and the parameters of a particular model. This allows one to disambiguate between different connectivity architectures that may underlie induced responses and to make quantitative inferences about the coupling among distributed cortical regions. Furthermore, one can assess changes in coupling that result from experimental manipulations or pathophysiology. Critically, this model allows one to distinguish between changes in linear and non-linear coupling in the brain. This work represents a further extension of dynamic causal modelling to cover spectral responses as measured by the EEG or MEG (David et al., 2006a; Kiebel et al., 2006; Moran et al., 2007).

There are many approaches to detecting and estimating neuronal coupling using frequency-based analyses of electrophysiological recordings. These can be divided into descriptive and 
mechanistic; a distinction that is closely related to the difference between functional and effective connectivity. In the following, we discuss various approaches to motivate the present model. In contrast to dynamic causal modelling, the majority of current approaches are descriptive in nature:

\section{Descriptive approaches: detecting functional connectivity}

Functional connectivity has been defined as the statistical dependence among remote neurophysiological time-series. To establish functional connectivity one has to show that the statistical dependencies are significant. This entails, in its most general formulation, measuring the mutual information among two or more time-series (Roulston, 1999; Quian Quiroga et al., 2002). There are several approaches to assessing mutual information, which divide broadly into linear and non-linear. The most common approach uses linear systems theory and measures the correlation or coherence between two time-series. It has been shown repeatedly that these measures (the information in the cross-correlation function and coherence is identical) are very useful for quantifying long-range interactions using EEG (Bressler, 1995; Gross et al., 2001; Nunez et al., 1997). Measures of linear dependencies can be generalised to multivariate time-series to furnish interesting formulations in terms of directed transfer functions and Granger causality (Brovelli et al., 2004; Bernasconi and Konig, 1999).

\section{Non-linear methods}

In terms of non-linear approaches; the most general approach relies upon the notion of generalised synchrony (Rulkov et al., 1995; Schiff et al., 1996), which posits a mapping between the manifolds containing the state-space trajectories of two time-series. These time-series may not be correlated or indeed have any obvious formal similarity in their periodic structure. These techniques usually rely upon some form of temporal embedding or attractor reconstruction. Generalised synchronisation exists between two dynamical systems when the state of the response system is a function of the state of the driving system. If this function is continuous, two neighbouring points on the attractor of one system should correspond to two nearby points on the attractor of the other. This correspondence is used to see if the evolution of neighbouring trajectories in one attractor can be used to predict the evolution of a point on the other attractor (see Breakspear and Terry (2002) for an example). Usually, generalised synchrony is used to detect nonlinear coupling by comparing the mutual predictability (information) between time-series before and after destroying their non-linear dependencies (by randomising their phase relationships). A special case of generalised synchrony is phase-synchrony.

Phase-synchronisation, between two oscillators, is a ubiquitous phenomenon, which appears when they are coupled in a broad range of structures, including EEG sources (Pikovsky et al., 2001). Timefrequency analysis of phase-synchronisation is popular in current research on cortical networks (David et al., 2003; Engel et al., 2001; Varela et al., 2001; Lee et al., 2003). Establishing phasesynchronisation proceeds in two steps; estimation of instantaneous phase and the quantification of the phase locking. This quantification uses the distribution of phase differences to establish significant mutual information between the two time-series. Both generalised and phase-synchrony can be expressed between coupled systems that show autonomous or indeed chaotic dynamics.

Another approach to detecting non-linear coupling is based on non-linear system identification theory for controllable systems.
This approach formulates dependencies in terms of generalised or non-linear transfer functions that are estimated using generalised or poly-spectral analysis. Bispectral measures such as bicoherence (Dumermuth et al., 1971) have been used to detect non-linear coupling in human EEG (Jeffrey and Chamoun, 1994; Shils et al., 1996). The key thing about non-linear coupling is that it induces dependencies among different frequencies. We will exploit this below (see also Friston (2000)). The same non-linear crossfrequency coupling is seen in phase-synchrony; two principal forms of cross-frequency phase interactions are recognized (Palva et al., 2005): n:m phase-synchrony, which indicates amplitudeindependent phase locking of $n$ cycles of one oscillation to $m$ cycles of another (Tass et al., 1998), and nested oscillations, which reflect the locking of the amplitude fluctuations of faster oscillations to the phase of a slower oscillation (Vanhatalo et al., 2004). These forms of phase-synchronisation can be used to disclose nonlinear coupling, in which a slower frequency comes to entrain or be entrained by a faster frequency.

In summary, there are several ways to establish the statistical dependencies between two measured time-series (see David et al. (2004) for a comparison of these approaches and Pereda et al. (2005) for a comprehensive overview of non-linear methods). However, they are all concerned principally with detecting functional connectivity; they are not concerned with the mechanisms or causes that underlie these dependencies.

\section{Modelling approaches: estimation of effective connectivity}

Effective connectivity is defined as the influence that one neural system exerts over another. Critically, this definition posits a causal mechanism for the dependencies described above. In a time-series setting, these models are usually dynamic and rest on differential equations that are causal in a control theory sense. We refer to these models as dynamic causal models (Friston et al., 2003; David et al., 2006b). The idea behind dynamic causal modelling is to explain observed responses in terms of a dynamic system that is perturbed by exogenous inputs that are either known or unknown. The model is defined by the form and parameters of differential equations that describe the evolution of the system states. Inversion of these models allows one to make inferences about the models and their parameters. Critically, this allows one to compare different models and quantify them in terms of the conditional density over both models and parameters. These models are based on specific hypotheses about putative sources and their assumed connectivity. This is a fundamental departure from descriptive approaches to functional connectivity because it allows one to answer questions about the mechanisms and functional architectures that cause observed responses. These questions are posed in terms of competing models, which are evaluated in relation to each other; clearly, the answers obtained are conditional on the models considered.

In contrast to the descriptive approaches, there are relatively few causal models of spectral responses. Those that do exist can, again, be divided into linear and non-linear. Linear models are normally derived by linearising a neurobiologically informed nonlinear model of neuronal dynamics (e.g., a mean-field or neuralmass model) and evaluating the spectral response under some assumptions about the spectral composition of exogenous input (Wright and Liley, 1994). Steady-state spectral measurements can then be used to invert the model and infer on important biophysical parameters such as rate constants or coupling parameters (Rowe et al., 2005; Moran et al., 2007). In the non-linear and dynamic 
domain there are even fewer models. An important class is models that are based upon loosely coupled oscillators:

\section{Models of spectral dynamics}

The theory of coupled phase oscillators has found many applications to biological, chemical and physical phenomena (Kuramoto, 1984; Kopell and Ermentrout, 1986). Under certain assumptions, the behaviour of networks of neurons with largely oscillatory output can be approximated by a system of equations that govern the phases of each oscillator (Ermentrout and Kleinfeld, 2001)

$\dot{\psi}_{i}=\omega+\sum_{j} f\left(\psi_{i}-\psi_{j}\right)$

Here $\psi_{i}$ is the instantaneous phase of the $i$-th unit or population; $\omega=2 \pi v$, where $v$ is the intrinsic frequency of the oscillators and $f$ $\left(\psi_{i}-\psi_{j}\right)$ is the effective coupling, which is a non-linear [periodic] function of the phase-difference between two oscillators. The sum runs over all units that are connected. In these models, it is assumed that the amplitude of the oscillations is unimportant and the key dynamics are narrow-band. Although this equation could be used as the basis of a dynamic causal model, it has not (to the best of our knowledge).

The model above speaks to a specific class of dynamic causal model (DCM): DCMs can be phenomenological or biophysical. Biophysical DCMs are constrained by the known physical or biological processes generating the observed signals. In contrast, phenomenological DCMs describe the causal dynamics in a purely formal fashion. Eq. (1) is an example of this, where the form of the effective coupling can be motivated using neurobiological constraints (see Ermentrout and Kleinfeld (2001)) but is not formulated explicitly in terms of neuronal processes. The DCM described in this paper is phenomenological and complements models based on instantaneous phase by modelling the evolution of instantaneous power:

$\dot{g}(\omega)_{i}=\sum_{j} f\left(g(\omega)_{i}, g(\omega)_{j}\right)$

Here, $g(\omega)_{i}$ is the spectral density, over frequency $\omega$, of the $i$-th unit. In this model, temporal changes of power in a source are explained as a network function of power in all sources. This sort of model can deal with situations in which oscillations in one area become amplitude modulated by oscillations in another band in the same or another areas; e.g., theta-band modulation of gamma activity (e.g., Guderian and Duzel, 2005). Here, high levels of theta activity would engender increases in gamma.

This model is phenomenological in the sense we make no attempt to motivate the form of effective coupling, $f\left(g_{i}, g_{j}\right)$ but simply use the coefficients of its Taylor expansion as parameters (see below). This is exactly the same device used in bilinear DCMs for functional magnetic resonance imaging (fMRI) time-series (Friston et al., 2003) and is recapitulated here for spectral responses as measured with EEG or MEG. The ensuing simple form for the DCM is particularly useful because it allows us to partition the effective coupling between regions at the same frequency and between regions across frequencies. This is important because, as noted above, within-frequency coupling is generally mediated by linear mechanisms, whereas cross-frequency coupling rests on non-linear in mechanisms. This is pertinent to neuronal dynamics, where nonlinear mechanisms may predominate in functional integration.

In summary, the DCM elaborated below describes the phenomenological evolution of spectral densities in multivariate time-series; it is formulated to model coupling within and between frequencies that are associated with linear and non-linear mechanisms respectively. This is similar to the bilinear form adopted for fMRI, which distinguishes between task or stimulus-invariant coupling (linear) and context-sensitive (non-linear or bilinear) changes in that coupling.

\section{Overview}

This paper comprises four sections. In the first, we reprise, briefly, a generalised convolution model of neuronal coupling (Friston, 2000) to demonstrate the link between cross-frequency coupling and non-linear mechanisms. We illustrate these phenomena using a neural-mass model that is the basis of biophysical DCMs for ERPs (David et al., 2006b). In the second section, we describe a DCM for induced responses and relate its parameterisation to the generalised convolution models of the first section. The model provides the likelihood function of a generative model, which is inverted using standard variational techniques. This inversion is summarized briefly in the last part of this section. In the third section, we try to establish the face validity of our model using synthetic data, where the true inputs and architecture are known. We generated synthetic data and compared linear and non-linear models to identify the veridical model. This enabled us to establish face validity and see how the inversion behaves under different noise levels. The fourth section provides a demonstration of the model and its inversion using real EEG data acquired during face perception.

\section{Non-linear and cross-frequency coupling}

In this section, we show why non-linear mechanisms are mandatory for coupling across frequencies. We have dealt with this issue in a series of papers on the theoretical neurobiology of functional integration (e.g., Friston, 1997, 2000, 2001) and summarize the main points here. The results in this section are not necessary to derive the dynamic causal model of the next section; they are used to highlight the sorts of behaviours that this model has to accommodate.

\section{Generalised convolution models}

In what follows, we treat any neuronal system or electromagnetic source as an input-state-output system. We will show that if this neuronal system is non-linear, the energy at one frequency in the inputs (from other sources) manifests at different frequencies in the outputs. This induces cross-frequency coupling between any two sources, when the output of one serves as the input the other. The Fliess fundamental formula (Fliess et al., 1983) describes the causal relationship between system outputs and the history of its inputs. This relationship conforms to a Volterra series, which expresses the output as a generalised convolution of the input, critically without reference to any hidden states. This series is a functional Taylor expansion of the outputs, $y(t)$ with respect to the inputs $u(t)$ (Bendat, 1990). The reason it is a functional expansion is that the inputs are a function of time. ${ }^{1}$

$$
\begin{aligned}
& y(t)=\sum_{i} \int \ldots \int \kappa_{i}\left(\sigma_{1}, \ldots, \sigma_{i}\right) u\left(t-\sigma_{1}\right), \ldots, u\left(t-\sigma_{i}\right) d \sigma_{1}, \ldots, d \sigma_{i} \\
& \kappa_{i}\left(\sigma_{1}, \ldots, \sigma_{i}\right)=\frac{\partial^{i} y(t)}{\partial u\left(t-\sigma_{1}\right), \ldots, \partial u\left(t-\sigma_{i}\right)}
\end{aligned}
$$

\footnotetext{
${ }^{1}$ For simplicity, we will deal with a single input and a single output.
} 
where $\kappa_{i}\left(\sigma_{1}, \ldots \sigma_{i}\right)$ is the $i$-th order kernel. The integrals are over the past or history of the inputs, which renders the system causal. Introducing the spectral representation in terms of the unitary Fourier transform pair $^{2}$

$$
\begin{aligned}
u(t) & =\int s_{u}(\omega) e^{j \omega t} d \omega \\
s_{u}(\omega) & =\int u(t) e^{-j \omega t} d t
\end{aligned}
$$

where, $g_{u}(\omega)=\left\langle s_{u}(\omega), s_{u}(-\omega)\right\rangle$ is spectral density; we can rewrite the Volterra expansion and it transform as

$y(t)=\sum_{i} \int_{-\pi}^{\pi} \ldots \int_{-\pi}^{\pi} \mathrm{e}^{j\left(\omega_{1}+, \ldots,+\omega_{i}\right) t} \Gamma_{i}\left(\omega_{1}, \ldots, \omega_{i}\right) s_{u}\left(\omega_{1}\right), \ldots, s_{u}\left(\omega_{i}\right) d \omega_{1}, \ldots, d \omega_{i}$
$s_{y}(\omega)=\sum_{i} \int_{-\pi}^{\pi} \ldots \int_{-\pi}^{\pi} \Gamma_{i}\left(\omega_{1}, \ldots, \omega_{i}\right) s_{u}\left(\omega_{1}\right), \ldots, s_{u}\left(\omega-\omega_{1} \ldots-\omega_{i}\right) d \omega_{1}, \ldots, d \omega_{i-1}$

where the functions

$$
\begin{gathered}
\Gamma 1\left(\omega_{1}\right)=\int_{0}^{\infty} \mathrm{e}^{-j \omega_{1} \sigma_{1}} \kappa_{1}\left(\sigma_{1}\right) d \sigma_{1} \\
\Gamma_{2}\left(\omega_{1}, \omega_{2}\right)=\int_{0}^{\infty} \int_{0}^{\infty} \mathrm{e}^{-j\left(\omega_{1} \sigma_{1}+\omega_{2} \sigma_{2}\right)} \kappa_{2}\left(\sigma_{1}, \sigma_{2}\right) d \sigma_{1} d \sigma_{2} \\
\ldots
\end{gathered}
$$

are the Fourier transforms of the kernels. These functions are called generalised transfer functions and mediate the expression of frequencies in the output given those in the input. Critically, the influence of higher order kernels, or equivalently generalised transfer functions means that a given frequency in the input can induce a different frequency in the output. A simple example of this would be squaring a sine-wave input to produce an output of twice the frequency (Friston, 2001). Generalised transfer functions are usually estimated through estimates of polyspectra. For example, the spectral form of Eq. (5) and its high-order counterparts are ${ }^{3}$

$$
\begin{aligned}
g_{u y}\left(-\omega_{1}\right) & =\Gamma_{1}\left(\omega_{1}\right) g_{u}\left(\omega_{1}\right) \\
g_{\text {uuy }}\left(-\omega_{1},-\omega_{2}\right) & =2 \Gamma_{2}\left(\omega_{1}, \omega_{2}\right) g_{u}\left(\omega_{1}\right) g_{u}\left(\omega_{2}\right) \\
& \vdots \\
g_{u \ldots y}\left(-\omega_{1}, \ldots,-\omega_{n}\right) & =n ! \Gamma_{n}\left(\omega_{1}, \ldots, \omega_{n}\right) g_{u}\left(\omega_{1}\right) \ldots g_{u}\left(\omega_{n}\right)
\end{aligned}
$$

Coherence (sometimes called coherency), $g_{u y}(\omega)$ is simply the second-order cross-spectrum between the input and output and is related to first-order effects (i.e., the first-order kernel or transfer function). Coherence is therefore a surrogate for first-order or linear connectivity. Bicoherence or the cross-bi-spectrum $g_{\text {uuy }}\left(\omega_{1}, \omega_{2}\right)$ is the third-order cross-poly-spectrum and implies a non-zero secondorder kernel or transfer function. See Friston (2000), Jeffrey and Chamoun (1994) and Shils et al (1996) for examples of detecting non-linear coupling with bi-spectral analyses and Priestley (1988) for the mathematical background.

In the present context, the thing we need to take from this formulation is that the only way one frequency in the input can modulate another frequency in the output is through second or higher-order kernels. This means that dependencies between different frequencies are mediated by non-linear coupling. We can express this in terms of

\footnotetext{
${ }^{2}$ Omitting constants of proportionality for clarity.

3 These equalities hold only when the Volterra expansion contains just the $n$-th order term and are a generalisation of the classical results for the transfer function of a linear system.
}

the changes in the spectral density of the response, induced by changes in the input; where, under linear coupling

$\frac{\partial g_{y}\left(\omega_{1}\right)}{\partial g_{u}\left(\omega_{2}\right)}=\left\{\begin{array}{cc}\left|\Gamma_{1}\left(\omega_{1}\right)\right|^{2} & \omega_{1}=\omega_{2} \\ 0 & \omega_{1} \neq \omega_{2}\end{array}\right.$.

\section{An illustration using a non-linear neural-mass model}

To illustrate the induction of responses across different frequency bands, we evaluated the time-frequency power of the input and response of a non-linear neural-mass model of electromagnetic sources (Jansen and Rit, 1995). The neural-mass model is the same model used in David et al. (2006b) and is used in DCM for event-related potentials.

Briefly, the model is based on linear post-synaptic responses to pre-synaptic input. Three different populations are coupled using their mean firing rates, which are a static non-linear function of voltage as shown in Fig. 1. The dynamics of each neuronal subpopulation $i$ are governed by second-order differential equations in voltage of the form

$\ddot{V}_{i}+2 \kappa_{i} \dot{V}_{i}+\kappa_{i}^{2} V_{i}=\kappa_{i} H_{i} \sum_{j} \gamma_{i j} S\left(V_{j}\right)$.

The form of the implicit response kernel and non-linear voltagefiring curve, $S\left(V_{j}\right)$ is shown in Fig. 2. The three subpopulations correspond roughly to the supragranular, infragranular and granular layers of cortex and are interconnected (with coupling parameters, $\gamma_{i j}$ ) according to known connectivity rules. The non-linearity in Eq. (8) makes this a useful model of weak non-linear coupling among neuronal sources (Jansen and Rit, 1995). Figs. 3 and 4 such show the results of perturbing this model of neural masses; the time-frequency profile of the input and responses shows how non-linear transformations induce frequencies not present in the input:

The top panels of Fig. 3 show the input (left) and output (right) in the time-domain and the lower panels show the same data in timefrequency format. The response at each time and frequency was estimated using a Fourier transform with a sliding Gaussian window

$g_{y}(\omega, t)=\left|\int y(s-t) \exp \left(-s^{2} / 2 \sigma^{2}\right) \mathrm{e}^{-j \omega s} d s\right|^{2}$

where $\sigma=256 \mathrm{~ms}$ specifies the width of the window. The input was a four second box-car function plus random fluctuations, sampled from a Gaussian density with a standard deviation of one sixteenth of the box-cars amplitude. It can be seen that the response has a very different spectral profile to the input, with marked power in the 10 $20 \mathrm{~Hz}$ range. However, this response could be meditated by linear effects and represent a filtering of the broad-band input. To illustrate non-linearity, we repeated the simulation but using a four second pure sine-wave input at $16 \mathrm{~Hz}$. The left-hand panels of Fig. 4 show clearly that this single frequency induces structured responses at much higher frequencies. To ensure that this cross-frequency induction was mediated by non-linear mechanisms, we repeated the simulation but scaled the input down by a factor of 128 ; this keeps neuronal states within the linear regime of the models depolarisation-firing curve and suppresses non-linearity (see Fig. 2, right). Following this single change, the spectral output is now a quasi-copy of the input (see right-hand panels). It is this sort of linear and non-linear neuronal coupling, among neuronal populations, we want to model and make inferences about.

In the next section, we describe our dynamic causal model for induced responses, where the states of each neuronal source are summarized in terms of their spectral profiles. We will see that a simple parameterisation of this model allows for a partitioning of within and between-frequency coupling and, implicitly, a partition- 


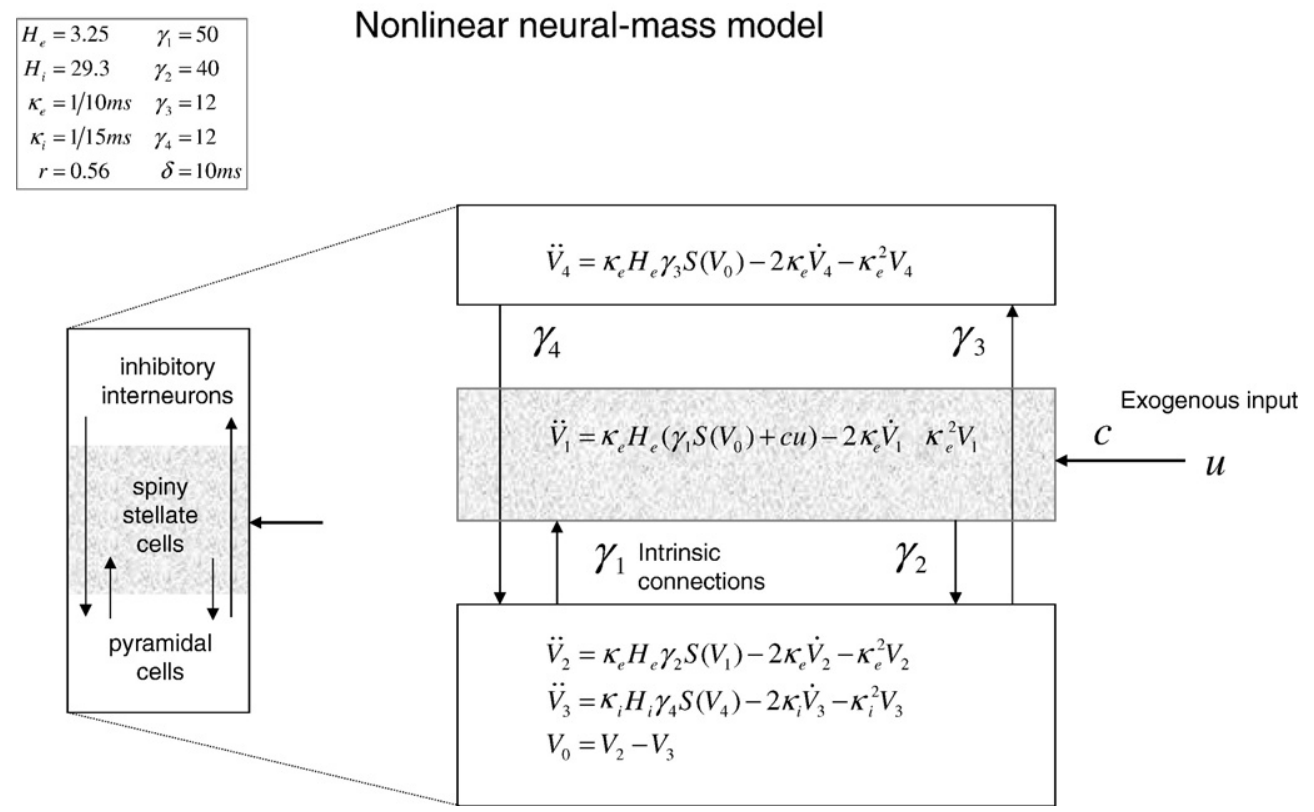

Fig. 1. Non-linear neural-mass model used to illustrate non-linear transformations (see David et al. (2003) for details). This model comprises three interconnected subpopulations. The model uses a transmembrane potential state-space model at the synaptic level and a non-linear sigmoid transformation $S(V)$ at the soma of neurons to model spike rates.

ing into dynamics that can be attributed to linear and non-linear effects.

\section{A dynamic causal model}

This section describes the dynamic causal model, which we will invert in subsequent sections to make inferences about synthetic and real data. Probabilistic model inversion requires a generative model. A probabilistic generative model requires the specification of a likelihood model and its priors. The likelihood model simply describes the probability of obtaining some data features (in our case spectra) given a model and its parameters, while the priors place constraints on the parameters.

$$
\kappa(t)_{e}=\kappa_{e} H_{e} t \exp \left(-\kappa_{e} t\right)
$$

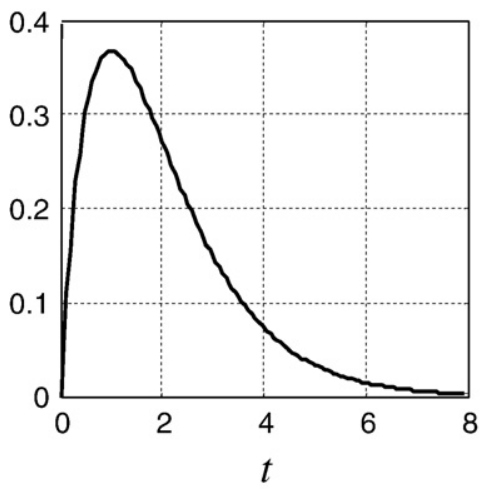

\section{Voltage response to synaptic input}

\section{A model for spectral features}

The generalised convolution model of the previous section assumes that neuronal dynamics are stationary; i.e., they express the same power over time. This model is fine for continuous steady-state electrophysiological recordings and has been used as a DCM for steady-state local field potential recordings (Moran et al., 2007). However, evoked brain dynamics are non-stationary and evolve over peristimulus time. This means we need a DCM of time-dependent changes in spectral energy. The model described below assumes that the spectral energy at one frequency in a source causes changes in the same (linear coupling) or different (nonlinear coupling) frequencies, in other sources. It is fairly simple to

$$
S(V)=\frac{1}{1+\exp (-r V)}-\frac{1}{2}
$$

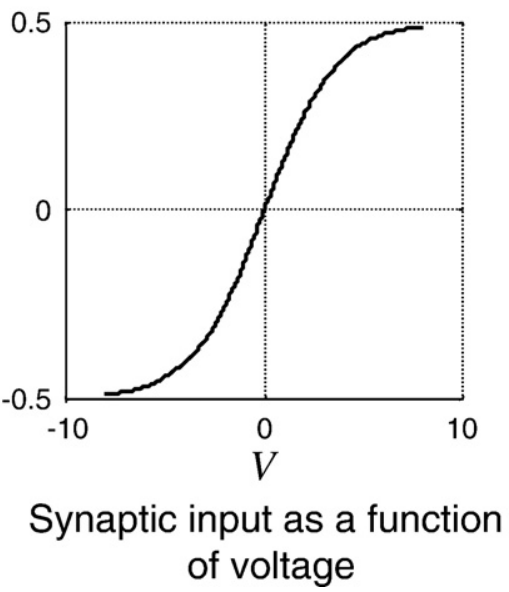

Fig. 2. The implicit form of the linear impulse response function of transmembrane potential (left) and the sigmoid firing-input curve $S(V)$ (right). 

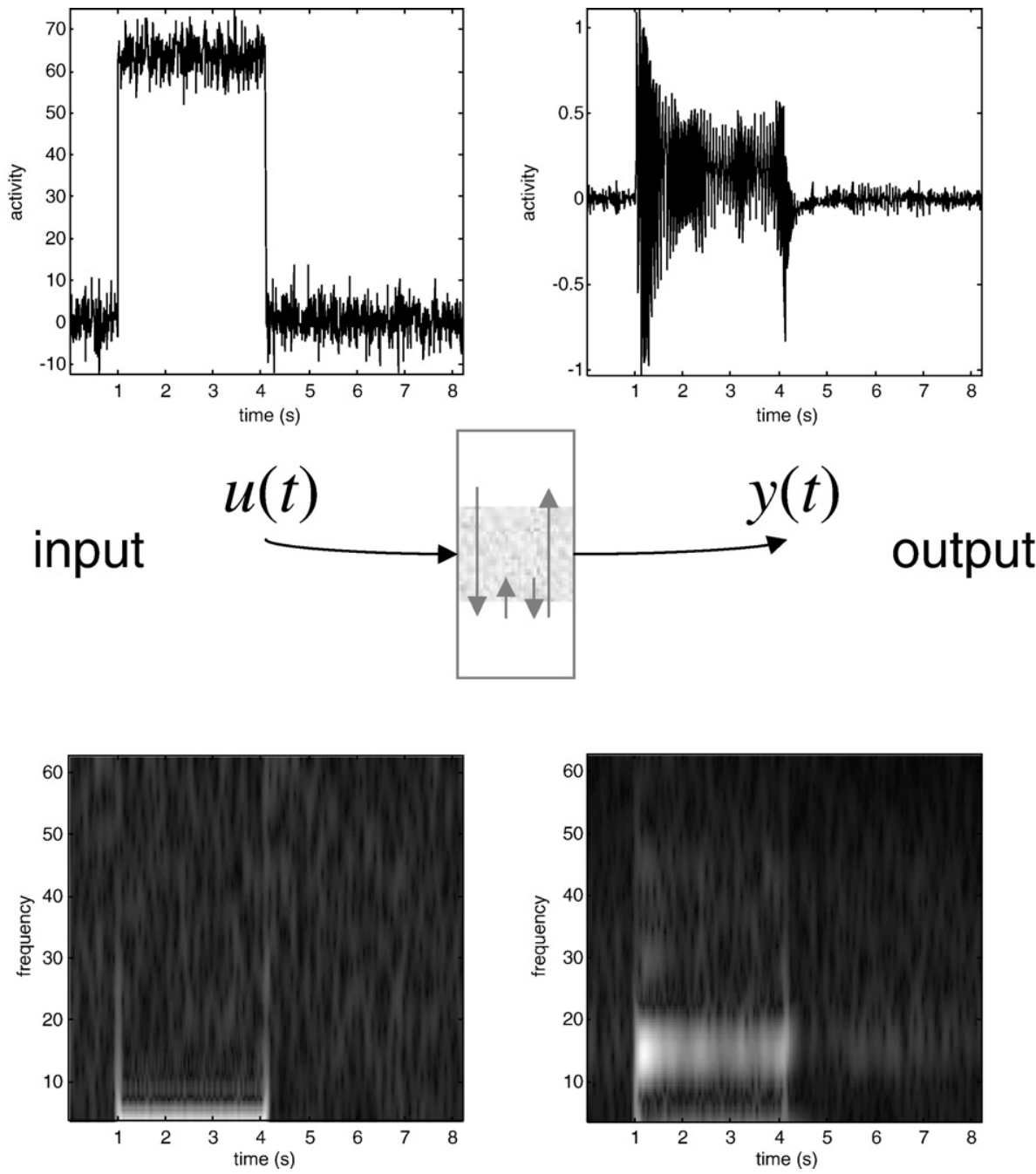

Fig. 3. The input-output relation of the neural-mass model (Fig. 1) in the time-frequency domain. Left: deterministic input comprising a four second box-car function plus random fluctuations (top) and its spectral profile (bottom). Right: response time course (top) and its spectral profile (bottom).

show that, under a linear state-space model of these changes in spectral density, the coupling between changes in frequency determines the coupling between frequencies at steady-state. This means that between-frequency coupling in the DCM must be mediated by non-linear mechanisms (by Eq. (7) of the previous section).

Consider $J$ sources in the brain, each described by a state vector $g_{j}(\omega, t) \in \mathfrak{R}^{K \times 1}$ of spectral densities at $K$ frequencies

$g_{j}(\omega, t)=\left[\begin{array}{c}g_{j}\left(\omega_{1}, t\right) \\ \vdots \\ g_{j}\left(\omega_{K}, t\right)\end{array}\right]$

We will treat these spectral states as perturbations around their expected levels, in the absence of exogenous input. We can model the dynamics of these spectral states using a first-order Taylor expansion of Eq. (2) to give

$$
\tau \dot{g}(t)=\tau\left[\begin{array}{c}
\dot{g}_{1} \\
\vdots \\
\dot{g}_{J}
\end{array}\right]=\left[\begin{array}{ccc}
A_{11} & \cdots & A_{1 J} \\
\vdots & \ddots & \vdots \\
A_{J 1} & \cdots & A_{J J}
\end{array}\right] g(t)+\left[\begin{array}{c}
C_{1} \\
\vdots \\
C_{J}
\end{array}\right] u(t)
$$

where the matrices $A$ and $C$ contain coupling parameters that control changes in spectral activity induced by other sources and exogenous (e.g., stimulus) inputs, $u(t)$

$A_{i j}=\left[\begin{array}{ccc}a_{i j}^{11} & \cdots & a_{i j}^{1 K} \\ \vdots & \ddots & \vdots \\ a_{i j}^{K 1} & \cdots & a_{i j}^{K K}\end{array}\right] \quad C_{i}=\left[\begin{array}{c}c_{i}^{1} \\ \vdots \\ c_{i}^{K}\end{array}\right]$

Under this model, the scalar $a_{i j}^{k l}$ encodes how changes in the $k$-th frequency in the $i$-th source depend on the $l$-th frequency in the $j$-th source. The leading diagonal elements are $a_{i i}^{k k}=-1$; this means that each frequency has an intrinsic tendency to decay or dissipate. Similarly, $c_{i}^{k}$ controls the frequency-specific influence of exogenous inputs on the $k$-th frequency in the $i$-th source. This enables within and between-frequency coupling within and between sources. In later work, we will generalise the above model, $\tau \dot{g}=A g+C u$ to a bilinear approximation, in which experimental effects $v$, (e.g., condition or trial-type under which the responses were elicited) can change the coupling. This involves the inclusion of a bilinear term in Eq. (11) to give

$\tau \dot{g}=(A+v B) g+C u$. 
High amplitude inputs (nonlinear)

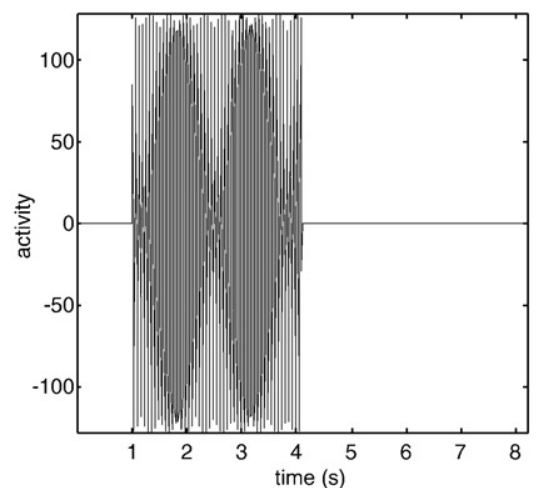

input

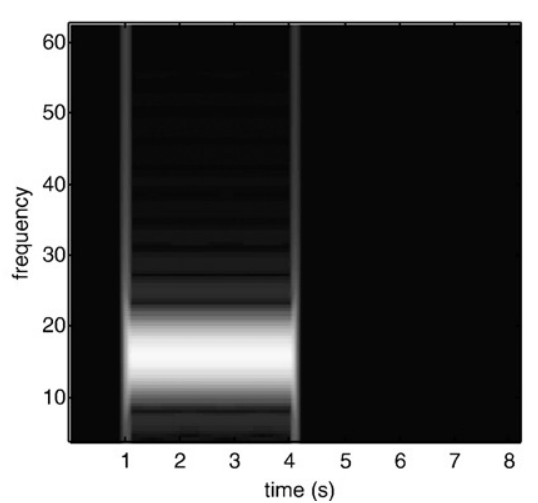

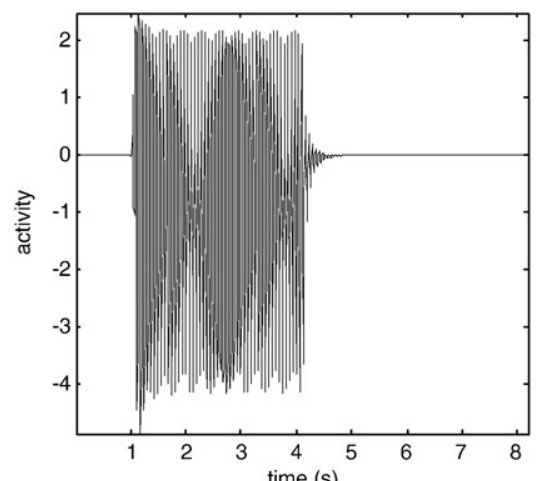

output

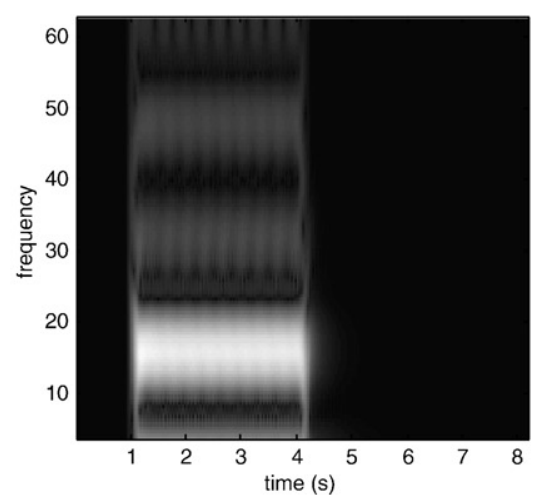

Low amplitude inputs (linear)

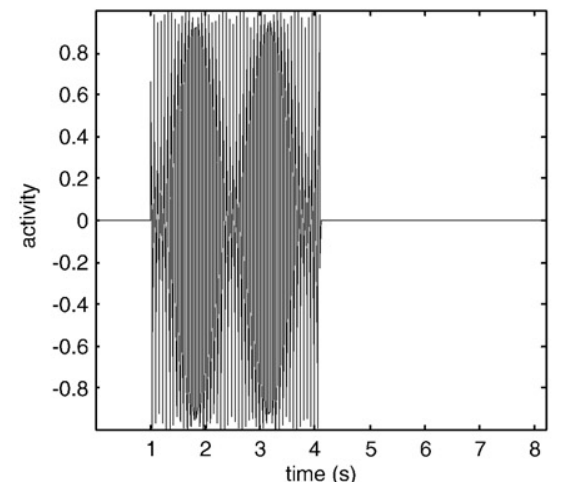

input

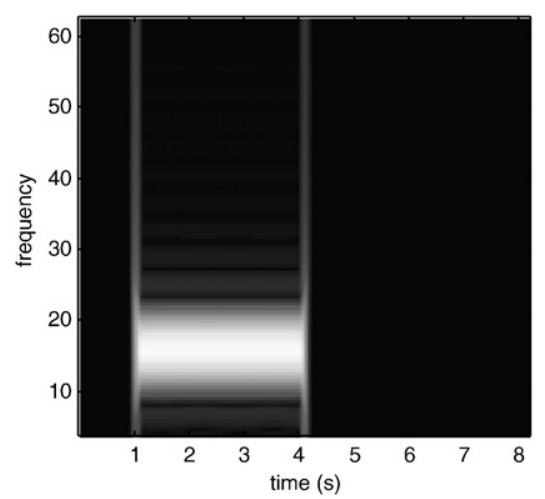

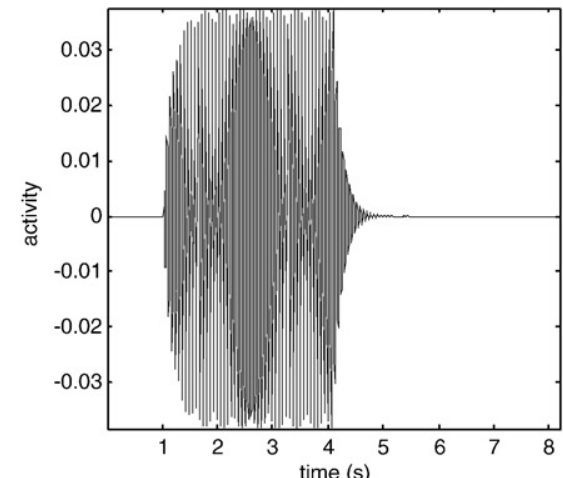

output

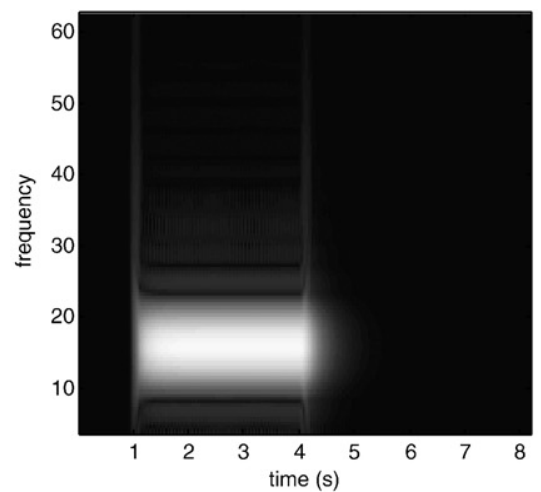

Fig. 4. The time-frequency profiles of inputs and responses of the neural-mass model in previous figures, showing non-linear and linear effects. Left panel: High input amplitude engages the non-linear regime of the

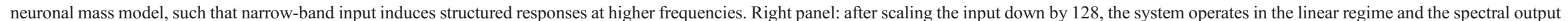
is a simple copy of the input. 
In this paper we will focus on the modelling of induced responses for a single trial-type and ignore trial-specific influences.

\section{Steady-states responses}

This simple first-order DCM can be related to the spectral representation of input-output systems of the preceding section by considering its equilibrium solution; in other words, the states to which the DCM converges. Under these steady-state conditions, $\dot{g}=0$ and $A g=-C u$. Recall that $a_{i i}^{k k}=-1$; this means that when the DCM is at equilibrium, the within-frequency coupling $a_{i j}^{k k}$ between sources plays the same role as the first-order transfer function of the previous section (c.f., Eq. (5)).

$$
\begin{aligned}
\dot{g}_{i}\left(\omega_{k}\right) & =0 \\
& =\sum_{j, l} a_{i j}^{k l} g_{j}\left(\omega_{l}\right)+c_{i}^{k} u \Rightarrow \\
g_{i}\left(\omega_{k}\right) & =\sum_{j, l \neq i, k} a_{i j}^{k l} g_{j}\left(\omega_{l}\right)+c_{i}^{k} u \\
& \Rightarrow \frac{\partial g_{i}\left(\omega_{k}\right)}{\partial g_{j}\left(\omega_{l}\right)}=\left\{\begin{array}{cc}
a_{i j}^{k k} & \omega_{k}=\omega_{l} \\
a_{i j}^{k l} & \omega_{k} \neq \omega_{l}
\end{array} .\right.
\end{aligned}
$$

Eq. (14) says that if the inputs are changing slowly (and we can assume $\dot{g} \approx 0$ ), we would see that long-term fluctuations in the $k$-th frequency in the $i$-th source scale with fluctuations in the $l$-th frequency of the $j$-th source in proportion to $a_{i j}^{k l}$. However, we know from the previous section that any dependencies among frequencies are mediated by non-linear mechanisms. This means that, under linear coupling, all the cross-frequency coupling parameters must be zero; $\forall i \neq j: a_{i j}^{k l}=0$. If any are not; $\exists i \neq j: a_{i j}^{k l} \neq 0$, we can infer a nonlinear coupling between sources $i$ and $j$. Clearly, we are not suggesting that steady-state is actually attained by the brain; but we can assume that convergence, following perturbation, is fast relative to changes in exogenous input. The rate of convergence can be arbitrarily fast, depending on the free parameter $\tau$ in Eq. (11), which is estimated from the data.

In summary, if we discount all the between-frequency couplings and consider the equilibrium solution of spectral dynamics (i.e., when the rate of change frequency is zero): the spectral power at a given frequency in one region is determined by the power at the same frequency in other regions. This is the sort of coupling that would be expected under linear mechanisms. Conversely, betweenfrequency coupling can be attributed to non-linear coupling.

\section{The spectral dynamics of sources}

Having established a model of spectral responses of the sources it is now necessary to specify how these responses are expressed in measurement space. In some instances this would not be necessary; for example, in local field potential or intracranial recordings obtained directly from each source. However, we will assume that the measurements have been obtained non-invasively using EEG or MEG. Consider the conventional linear forward model for electromagnetic sources $x(t)=\left[x_{1}(t), \ldots x_{J}(t)\right]^{T}$ and the corresponding lead-field matrix $L$ for multi-channel of data, $d(t)$. The observed response is a mixture of activity over all sources

$$
\begin{aligned}
d(t) & =L x(t) \Rightarrow \\
s_{d}(\omega) & =L s(\omega) \\
g_{d}(\omega) & =L\left\langle s(\omega) s(-\omega)^{T}\right\rangle L^{T}
\end{aligned} .
$$

This means that spectral responses in channel space are a mixture of the inner product of Fourier coefficients. Unfortunately, our DCM does not model these coefficients; this would require modelling both the power and phase of source activity, so that the coherence among sources $\left\langle s(\omega) s(-w)^{T}\right\rangle$ could be generated. To circumnavigate this problem, we project the data from channel space to the sources and then compute the spectral density

$$
\begin{aligned}
x(t) & =L^{-} d(t) \\
\tilde{g}_{i}(\omega, t) & =\left|\int x_{i}(s-t) \exp \left(-s^{2} / 2 \sigma^{2}\right) \mathrm{e}^{-j \omega s} d s\right|^{2}
\end{aligned}
$$

where $\tilde{g}(\omega, t)$ are the spectral responses modelled by our DCM and $L^{-}$is the generalised inverse of the lead-field matrix for our chosen sources. Eq. (16) is formally equivalent to a Morlet wavelet transform, where the window width scales inversely with the frequency. In our work we use $\sigma=k \omega^{-1}$, which covers about $k$ cycles; where $k>5$ is usually entertained in wavelet analyses.

This is unconventional for DCM, where one usually includes the spatial electromagnetic model within the DCM, so that both the neuronal and electromagnetic parts of the model are inverted together. In this application, we can regard the inversion of the electromagnetic part as feature selection, in the sense that Eq. (16) is a deterministic non-linear function of channel data that returns spectral features associated with specific source locations. The advantage is that there is a unique solution for the features because the prior specification of source locations means the inverse problem is not ill-posed; provided the number of (ECD) sources in small relative to the number of channels. Typically, the questions addressed by DCM are framed using, at most, eight sources. Note that the generalised inverse of the lead-field in Eq. (16) is one of many inversion schemes that one can use to project data from channel to source space (Darvas et al., 2004; Michel et al., 2004; Friston et al., 2008; Kiebel et al., 2008). The generalised inverse is an appropriate projector if one knows a priori where the sources are located. In other words, when there is no source localisation problem. Several colleagues have asked about extracting spectral features using beam-formers (Singh et al 2003). This would be a useful strategy if one did not know where the spectral signals were coming from. Beam-formers could then be used to localise the sources of predominant spectral dynamics. However, once these locations have been established, the generalised inverse of the

\begin{tabular}{|c|c|c|c|}
\hline Parameter $\theta$ & Description & Prior density $p(\theta)$ & \\
\hline $\begin{array}{l}\alpha=\theta_{\mu}^{2} / \theta_{\sigma} \\
\beta=\theta_{\mu} / \theta_{\sigma} \\
\theta_{\mu}=80 \exp \left(\theta_{1}\right) \\
\theta_{\mu}=1024 \exp \left(\theta_{2}\right)\end{array}$ & $\begin{array}{l}\text { Input (Gamma) } \\
\text { parameter }\end{array}$ & $\begin{array}{l}p\left(\theta_{1}\right)=N\left(0, \frac{1}{16}\right) \\
p\left(\theta_{2}\right)=N\left(0, \frac{1}{16}\right)\end{array}$ & \\
\hline$\tau=1 / 16 \exp (\kappa)$ & $\begin{array}{l}\text { Convergence } \\
\text { time }\end{array}$ & $p(\kappa)=N\left(0, \frac{1}{4}\right)$ & present \\
\hline$A_{i j}^{k l}$ & $\begin{array}{l}\text { Internal } \\
\text { coupling }\end{array}$ & $p\left(A_{i j}^{k l}\right)=\left\{\begin{array}{c}N(0,0) \\
N(-1,0)\end{array}\right.$ & $\begin{array}{c}\text { absent } \\
i=j \wedge k=l\end{array}$ \\
\hline$C_{i}^{k}$ & $\begin{array}{l}\text { External } \\
\text { coupling }\end{array}$ & $p\left(C_{i}^{k}\right)=\left\{\begin{array}{c}N\left(0, \frac{1}{16}\right) \\
N(0,0)\end{array}\right.$ & $\begin{array}{c}i=\text { input } \\
i \neq \text { input }\end{array}$ \\
\hline$G_{i}=\exp \left(\theta_{i}^{G}\right)$ & Power scaling & $p\left(\theta_{i}^{G}\right)=N\left(0, \frac{1}{512}\right)$ & \\
\hline
\end{tabular}
associated lead-field matrix furnishes a near-optimum ECD summary of activity that avoids suppression of local correlated activity. In this paper, we assume that the source locations are specified and leave optimisation of these spatial parameters to another paper.

Note that the model generates time-varying power at each source, whereas the spectral features we extract in Eq. (16) have

Table 1

Priors on model parameters 


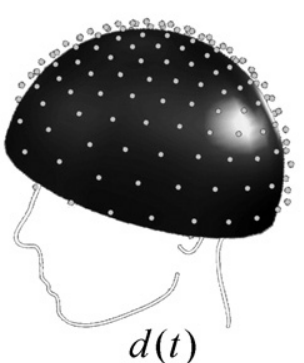

Data in channel space
Inversion of electromagnetic model $L$
$x(t)=L^{-} d(t)$

$\widetilde{g}_{j}(t)=U^{T}\left|F T\left(x_{j}(t)\right)\right|^{2}=U^{T}\left[\begin{array}{c}\widetilde{g}_{j}\left(\omega_{1}, t\right) \\ \vdots \\ \tilde{g}_{j}\left(\omega_{K}, t\right)\end{array}\right]$

$K$ frequency modes in $j$-th source

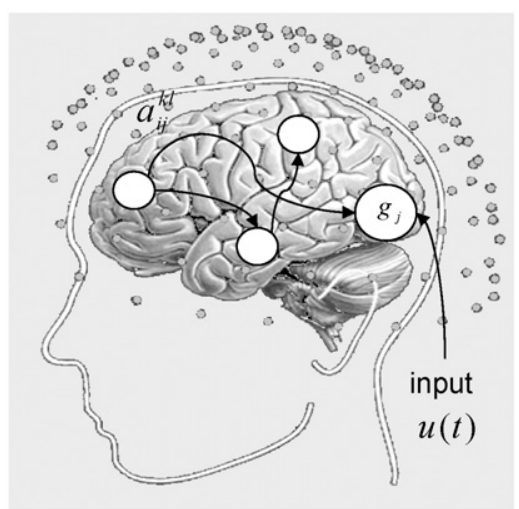

Neuronal model for spectral features

$$
\begin{gathered}
\text { Intrinsic (within-source) coupling } \\
\tau \dot{g}(t)=\tau\left[\begin{array}{c}
\dot{g}_{1} \\
\vdots \\
\dot{g}_{J}
\end{array}\right]=\left[\begin{array}{ccc}
A_{11} & \ldots & A_{1 J} \\
\vdots & \ddots & \vdots \\
A_{J 1} & \ldots & A_{J J}
\end{array}\right] g(t)+\left[\begin{array}{c}
C_{1} \\
\vdots \\
C_{J}
\end{array}\right] u(t) \\
\text { Extrinsic (between-source) coupling }
\end{gathered}
$$

Linear (within-frequency) coupling

$$
A_{i j}=\left[\begin{array}{ccc}
a_{i j}^{11} & \cdots & a_{i j}^{1 K} \\
\vdots & \ddots & \vdots \\
a_{i j}^{K 1} & \cdots & a_{i j}^{K K}
\end{array}\right]
$$

Nonlinear (between-frequency) coupling

Fig. 5. Schematic illustration of the analysis procedure. Upper panel: The spectral dynamics in the sources, $\tilde{g}_{j}(t)$, are first evaluated from observations in sensor space; they are projected onto source space using the pseudo-inverse of the lead-field, $L^{-}$. The spectral densities obtain by squaring the absolute values after Morlet wavelet transform. Lower panel: the linear form of state equations. At the neuronal level, the DCM comprises a vector of states for each electromagnetic source, allowing for linear and non-linear coupling.

three moments. It would be simple to include free parameters that map the predicted source power to these three moments but these parameters are of no interest. Therefore, we simply add the power over the moments and estimate a single free parameter, $G_{i}$, which scales the power of underlying neuronal dynamics to give the observed mixture $\tilde{g}_{i}(\omega, t)$.

\section{The probabilistic model}

We now have, under Gaussian assumptions about observation error, $\varepsilon$, a likelihood model for observed spectral activity in sources $\tilde{g}_{i}\left(\omega_{j}, t_{k}\right)$ that can be expressed as a mixture of predicted activity $g_{i}\left(\omega_{j}, t_{k}\right)$, baseline power and random fluctuations:

$$
\begin{aligned}
\tilde{g}_{i}\left(\omega_{j}, t_{k}\right) & =G_{i} g_{i}\left(\omega_{j}, t_{k}\right)+r_{i j}+\varepsilon_{i j k} \Leftrightarrow p\left(\tilde{g}_{i}^{j}\left(t_{k}\right) \mid \theta\right) \\
& =N\left(G_{i} g_{i}^{j}\left(t_{k}\right)+r_{i j}, \lambda^{-1} V\right) .
\end{aligned}
$$

The predicted activity was obtained by integrating Eq. (11), given the parameters, $\theta \supset \tau, A, C, G$ and input $u(t)$. The scalar $\gamma_{i j}$ models baseline power over time at the $i$-th source and $j$-th frequency. ${ }^{4}$ A likelihood model furnishes a way of measuring the likelihood of observed data; put simply, one generates a prediction using the model parameters and input. The probability of getting the observed data features is then specified by the amplitude of the prediction errors, relative to the precision (inverse variance) of the random fluctuations. $\lambda$ is the precision of this measurement noise in

\footnotetext{
${ }^{4}$ In practice, we estimate the baseline power as the frequency at the first time-bin.
}

feature-space (power over sources and frequency) and is estimated as a free parameter. This scale parameter scales a temporal correlation matrix $V$ encoding serial correlations among the observation noise. Because the time-frequency analysis necessarily smoothes random effects, we made $V$ a Gaussian autocorrelation matrix, with a standard deviation of $32 \mathrm{~ms}$. The standard deviation of the noise autocorrelation $\sigma_{V}>\sigma$ is bound by the window width, $\sigma$ in the timefrequency analysis in Eq. (16). This window imposes serial correlations on spectral data features and implicitly any random fluctuations. We chose a value that corresponds to the correlations induced by evaluating frequencies at $f \approx \sigma_{V}^{-1} \approx 30 \mathrm{~Hz}$.

In this work, the priors $p(\theta)$ on the model parameters were Gaussian shrinkage priors. Table 1 lists their prior densities and Fig. 5 provides a graphical summary of the ensuing model. Note that non-negative scale parameters have log-normal priors. ${ }^{5}$

\section{Exogenous input}

The predicted power was obtained by integrating Eq. (11). This requires the stimulus input to be specified. This exogenous input causes a burst of power in the network of sources. The frequency selectivity of this perturbation is encoded by the free parameters $C$ above. This input models changes in source activity, caused by putative subcortical afferents whose activities are time-locked to stimuli. The frequencies induced depend on the model's free parameters, which are optimised during inversion. From the point of view of each source, there is no real difference between the effects of

\footnotetext{
${ }^{5}$ We also used weakly informative log-normal hyperpriors on the precision hyperparameter.
} 
exogenous input and input from other sources (see Eq. (11)). Typically, only a few sources are allowed to receive exogenous input, which can have an arbitrary and source-specific frequency profile. Sources that do not receive input have their input parameters 'switched off' by priors that are precisely zero. The temporal form of the input is not known and has to be estimated. In this paper, we use a simple parameterisation, which assumes that the spectral perturbation has the form of a gamma distribution.

$u(t)=\frac{\beta^{\alpha} t^{\alpha-1} e^{-\beta t}}{\Gamma(\alpha)}$

where $\Gamma(\alpha)$ is the gamma function and priors on the input parameters, $\alpha, \beta$ are chosen such that the peak of the input is at about $80 \mathrm{~ms}$, with a dispersion of about $32 \mathrm{~ms}$ (see Table 1). The free parameters now comprise, $\theta \supseteq \alpha, \beta, \tau, A, C, G$.

The choice of $80 \mathrm{~ms}$ as the prior latency of the input is motivated by known latencies from single-unit electrode recording studies of visual and inferotemporal cortex (e.g., Hirsch et al., 2002). It takes this amount of time for visual input to reach secondary and higher visual areas. This is also the time when evoked sensory potentials start to express themselves in cortical sources (i.e., the N1 component). Note that the latency is a free parameter, so that suboptimal priors (within some reasonable bound), will be corrected during model inversion.

\section{Frequency bands and modes}

Hitherto, we have considered the states as spectral densities at a discrete number of frequencies or frequency bands. These states can be regarded as the coefficients of narrow-band spectral basis functions or frequency modes. In practice, we actually use the orthonormal principal modes of the source data, $U=\left[U_{1}, \ldots, U_{K}\right]$, obtained by a singular value decomposition of the spectral responses over time and sources, where $\tilde{g}=U \Lambda V^{T}$ and $\Lambda$ is a leading diagonal matrix of singular values. This means that instead of working with $K^{\prime}$ frequencies, we can reduce the problem to modelling the coupling among $K<K^{\prime}$ modes that cover all frequencies in different proportions.

In this context, the states $g_{i}^{k}$ represent the contribution of the $k$-th frequency mode, $U_{k}(\omega)$ to the spectral dynamics of the $i$-th region. We can project the predicted spectral dynamics in the state-space of frequency modes to frequency space using, $g_{i}(\omega)=U g_{i}$. Similarly, one can characterise the coupling as functions of frequency; i.e., $A_{i j}\left(\omega_{k}, \omega_{l}\right)=U A_{i j} U^{T}$ and $C_{i}(\omega)=U C_{i}$. These projections are possible because the frequency modes are orthonormal and we are using a linear DCM. We typically use between two and four modes, which account for about $90 \%$ of the observed variance in spectral responses. We have specified the likelihood and priors of our generative model and can now turn to model inversion and comparison.

\section{Bayesian inversion of DCMs}

In this section, we review briefly model inversion and selection. For a given DCM, say model $m$; parameter estimation corresponds to approximating the moments of the conditional or posterior distribution given by Bayes rule

$p(\theta \mid \tilde{g}, m)=\frac{p(\tilde{g} \mid \theta, m) p(\theta, m)}{p(\tilde{g} \mid m)}$.

The estimation procedure employed in DCM is described in Friston et al. (2006). The posterior moments (conditional mean $\mu$ and covariance $\Sigma$ ) are updated iteratively using Variational Bayes under a fixed-form Laplace (i.e., Gaussian) approximation to the conditional density $q(\theta)=N(\mu, \Sigma)$. This is equivalent to Expectation-Maximization (EM) that employs a local linearisation of the predicted responses about the current conditional expectation of the parameters. The $\mathbf{E}$-step conforms to a Fisher-scoring scheme that optimises the variational free energy $F(q, \lambda, m)$ with respect to the conditional moments. In the $\mathbf{M}$-step, the precision parameters $\lambda$ are updated in exactly the same way to provide their maximum likelihood estimates. The estimation scheme can be summarized as follows:

$$
\begin{aligned}
& \text { E-step } \quad q \leftarrow \max _{q} F(q, \lambda, m) \\
& \text { M-step } \quad \lambda \leftarrow \max _{\lambda} F(q, \lambda, m) \\
& \begin{aligned}
F(q, \lambda, m) & =\langle\ln p(\tilde{g} \mid \theta, \lambda)+\ln p(\theta)-\ln q(\theta)\rangle_{q} \\
& =\ln p(\tilde{g} \mid \lambda)-D(q \| p(\theta \mid \tilde{g}, \lambda))
\end{aligned}
\end{aligned}
$$

The free energy is simply a function of the log-likelihood and the log-prior and $q(\theta)$, which is an approximation to the posterior density $p(\theta \mid \tilde{g}, \lambda, m)$ we require. The last line of Eq. (20) shows that the free energy is the log-evidence or marginal likelihood minus the Kullback-Leibler divergence between the real and approximate conditional density. This means that the variational parameters (conditional moments and precision) maximize the log-evidence, while minimising the discrepancy between the true and approximate conditional density. This scheme is identical to that employed by DCM for fMRI and ERP.

\section{Model comparison and selection}

Inference on the parameters of a particular model uses the conditional density, $q(\theta)$. Usually, this involves specifying a parameter or compound of parameters as a contrast, $c^{T} \mu$. Inferences about this contrast are made using its conditional covariance, $c^{T} \Sigma(\lambda) c$. For example, one can compute the probability that a contrast is greater than zero. This inference is conditioned on the particular model specified. However, in many situations one wants to compare different models, for example models with and without particular connections. This entails Bayesian model comparison. Different models are compared using their evidence (Penny et al., 2004). The model evidence or marginal likelihood is

$p(\tilde{g} \mid m)=\int p(\tilde{g} \mid \theta, m) p(\theta \mid m) d \theta$.

The evidence can be decomposed into two components: an accuracy term, which quantifies the data fit, and a complexity term, which penalizes models with a large number of parameters. In the following, we approximate the model evidence for model $m$, with its free energy bound. After convergence, the divergence above is minimised and this bound become tight such that

$\ln p(\tilde{g} \mid m) \approx F(m)$.

The most likely model is the one with the largest log-evidence. This enables Bayesian model selection. Model comparison rests on the likelihood ratio of the evidence for two models. This ratio is the Bayes factor $B_{i j}$. For models $i$ and $j$

$\ln B_{i j}=\ln \frac{p(\tilde{g} \mid m=i)}{p(\tilde{g} \mid m=j)}=F(m=i)-F(m=j)$.

Conventionally, strong evidence in favour of one model requires the difference in log-evidence to be about three or more (i.e., a 


$$
U A_{i j} U^{T}=\sum_{k} \sum_{l} a_{i j}^{k l} U_{k} U_{l}^{T}
$$

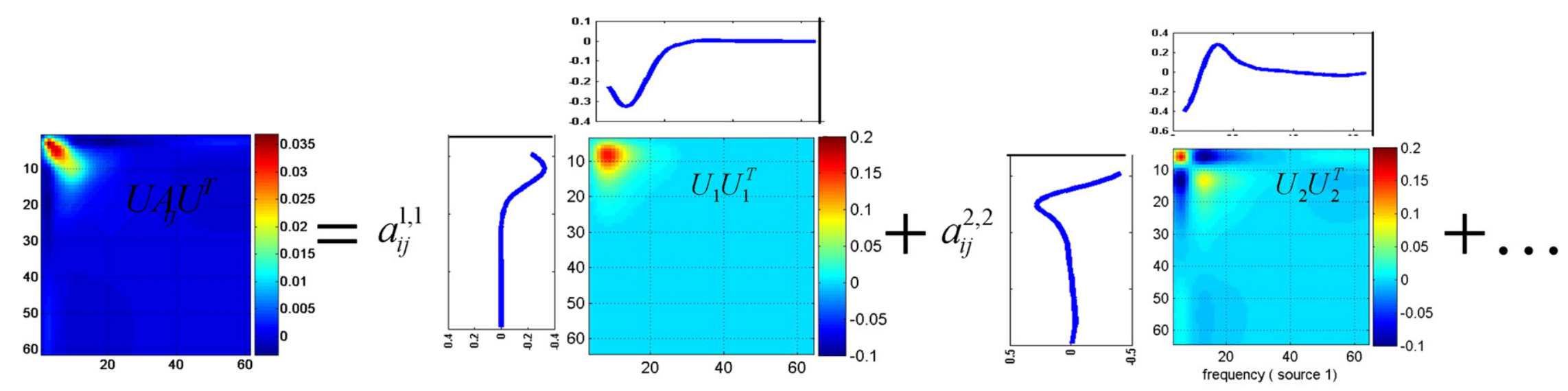

An example of linear (within-mode) coupling
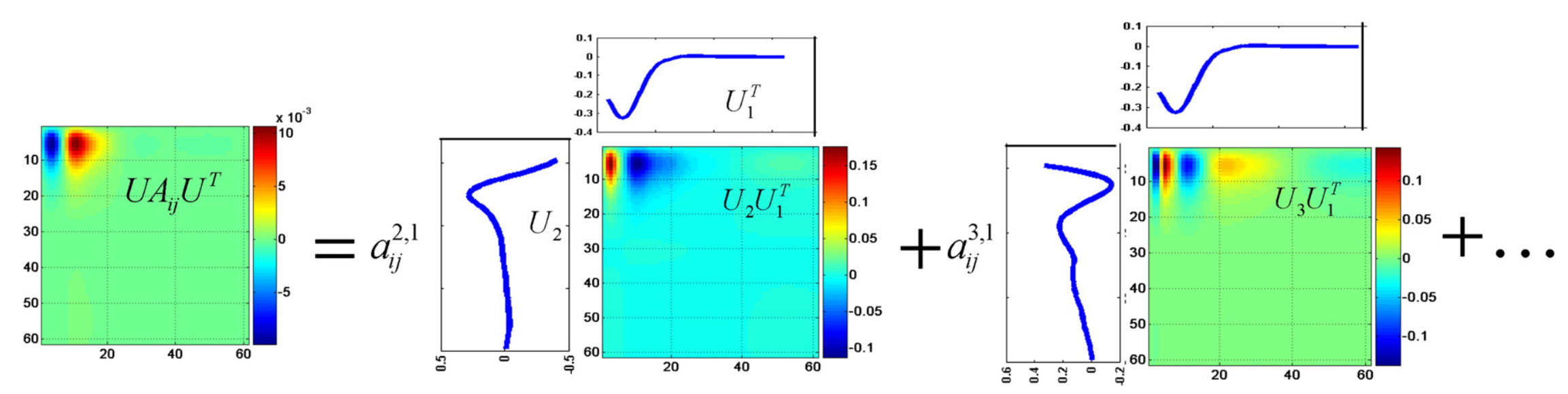

An example of nonlinear (between-mode) coupling

Fig. 6. Visualizing the estimated coupling parameters. These images represent the coupling strength between different frequencies and obtain by projecting the coupling matrices in mode-space to frequency space. Upper panels: linear components; lower panel: non-linear components; we will use this form in subsequent figures. 
relative probability of about twenty). In what follows, we will use the model comparison to compare models with and without various sorts of connections. By assuming uniform priors on the models we can convert the model evidence into a conditional probability over models by normalising the evidences so that they sum to one. Under this assumption, two models with a log-evidence of three imply that we can be $95 \%$ confident that the better model is more likely, given the data features.

\section{Summary}

Fig. 5 summarizes DCM for induced responses, which entails two steps. The first is to specify the model; i.e., the source locations and the network, based on prior knowledge or beliefs about the functional anatomy of the paradigm. For source locations, one can employ conventional source-reconstruction methods; for example, source imaging (Mattout et al., 2006) or equivalent current dipole (ECD) models (Kiebel et al., 2006, 2008). In terms of the network architecture, one needs to specify whether directed connections exist and whether they are linear or non-linear (i.e., whether the crossfrequency terms in are, a priori precisely zero or not).

The second step is to invert the model given some observed spectral features. The source spectra were obtained by projecting the channel data, $d(t)$, to source space and evaluating the spectral density over $K^{\prime}$ frequency bins using Eq. (16). These spectral features are reduced in number, using orthonormal frequency modes $U=\left[U_{i}, \ldots, U_{K}\right]$ to encode spectral dynamics. These dynamics are modelled using linear state equations (Eq. (14)), where the elements of the coupling matrix $a_{i j}^{k l}$ comprise the within $(i=j)$ and between $(i \neq j)$ source coupling parameters, which can be either within $(k=l)$ or between $(k \neq l)$ frequencies. The coupling between two regions can then be characterised as a function of source and target frequencies; $A_{i j}\left(\omega_{k}, \omega_{l}\right)=U A_{i j} U^{T}$ and displayed as a matrix or image. Linear coupling matrices have strong coupling among the same frequencies so that large coupling values are deployed along the leading diagonal. Conversely, non-linear coupling entails between-frequency effects with large off-diagonal terms (see Fig. 6). Because we use a small number of frequency modes, these coupling matrices encode broad-band coupling among the modes. This means that linear coupling can 'diffuse' away from the leading diagonal but retains its symmetrical form.

The above procedure can be repeated for several models or hypotheses about the underling architecture generating induced activity and the competing models compared using their differences in log-evidence.

We have now covered the specification, estimation and comparison of DCMs for induced responses. In the next section we try to establish their validity using synthetic data.

\section{Simulations}

This section addresses the face validity of the DCM described in the previous section. First we generated synthetic data to show that, using model comparison, the scheme can disambiguate competing models correctly. We use a very simple example to demonstrate the basic features of model selection. In the second simulations we used a more realistic model (based on the analysis of real data in the next section) to establish the identifiability of various parameters and ensure they can be estimated accurately under typical levels of noise. In all simulations, data were generated by integrating Eq. (11) given known model parameters (which also specify exogenous input). We then added noise to create synthetic data that were generated by a known architecture and known parameters. Critically, we used parameters that were based on the estimates from the analysis of real EEG data. This ensured that the simulations were biologically plausible. Observation noise was created by evaluating the timefrequency power of a white noise process, using the same wavelet transform employed in the empirical analyses. This ensured the serial correlations in the noise matched those observed empirically. Simulated noise processes were scaled and mixed with synthetic signal to give the desired signal-to-noise ratio (SNR).

\section{Model selection: distinguishing between linear and non-linear coupling}

In these simulations, we generated data using a very simple DCM under linear and non-linear coupling, with a SNR of $19.46 \mathrm{~dB}$. The model comprised two sources with two frequency modes in each source, where the first source projected to the second. See Table 2 for the values of the coupling parameters used (other parameters were set to their prior expectations in Table 1). The input to the system was a bump function that elicited responses in both modes in, and only in, the first region. The first DCM modelled all the connections as linear. The second used the same coupling parameters but allowed for fairly weak non-linear coupling from the second to the first frequency mode. We used two DCMs to invert these two data sets: The first modelled linear coupling only and the second allowed for non-linear coupling between the two sources. The model parameters and results of Bayesian inversion of these two simulated datasets are

\section{Table 2}

First simulations: results of inverting a linear and non-linear model using linear and non-linear data $(\mathrm{SNR}=19.46 \mathrm{~dB})$

\begin{tabular}{|c|c|c|}
\hline $\begin{array}{c}\text { True } \\
\text { parameter }\end{array}$ & $\begin{array}{l}A_{2,1}^{1,1}=0.72 \\
A_{2,1}^{2,2}=-0.45 \\
A_{2,1}^{2,1}=0.24\end{array}$ & $\begin{array}{l}A_{2,1}^{1,1}=0.72 \\
A_{2,1}^{2,2}=-0.45\end{array}$ \\
\hline $\begin{array}{c}\text { Non-linear } \\
\text { DCM }\end{array}$ & $\begin{array}{l}A_{2,1}^{1,1}=0.82 \quad p=0.99 \\
A_{2,1}^{2,2}=-0.45 \quad p=0.99 \\
A_{2,1}^{2,1}=0.32 \quad p=0.99 \\
F=-2561\end{array}$ & $\begin{array}{ll}A_{2,1}^{1,1}=0.72 & p=0.99 \\
A_{2,1}^{2,2} & \\
A_{2,1}^{2,1}=0.03 & p=0.96 \\
F=-4420\end{array}$ \\
\hline $\begin{array}{l}\text { Linear } \\
\text { DCM }\end{array}$ & $\begin{array}{c}A_{2,1}^{1,1}=1.3 \quad p=0.79 \\
A_{2,1}^{2,2}=-0.19 \quad p=0.99 \\
F=-2687\end{array}$ & $\begin{array}{c}A_{2,1}^{1,1}=0.76 \quad p=1.00 \\
A_{2,1}^{2,2}=-0.29 \quad p=0.99 \\
F=-4354\end{array}$ \\
\hline
\end{tabular}

$\mathrm{p}(A)$ indicates the conditional probability that the coupling parameter is greater than zero. $F$ is the log-evidence of each model and data pair. The winning model for each data set is indicated by a grey box. 
a
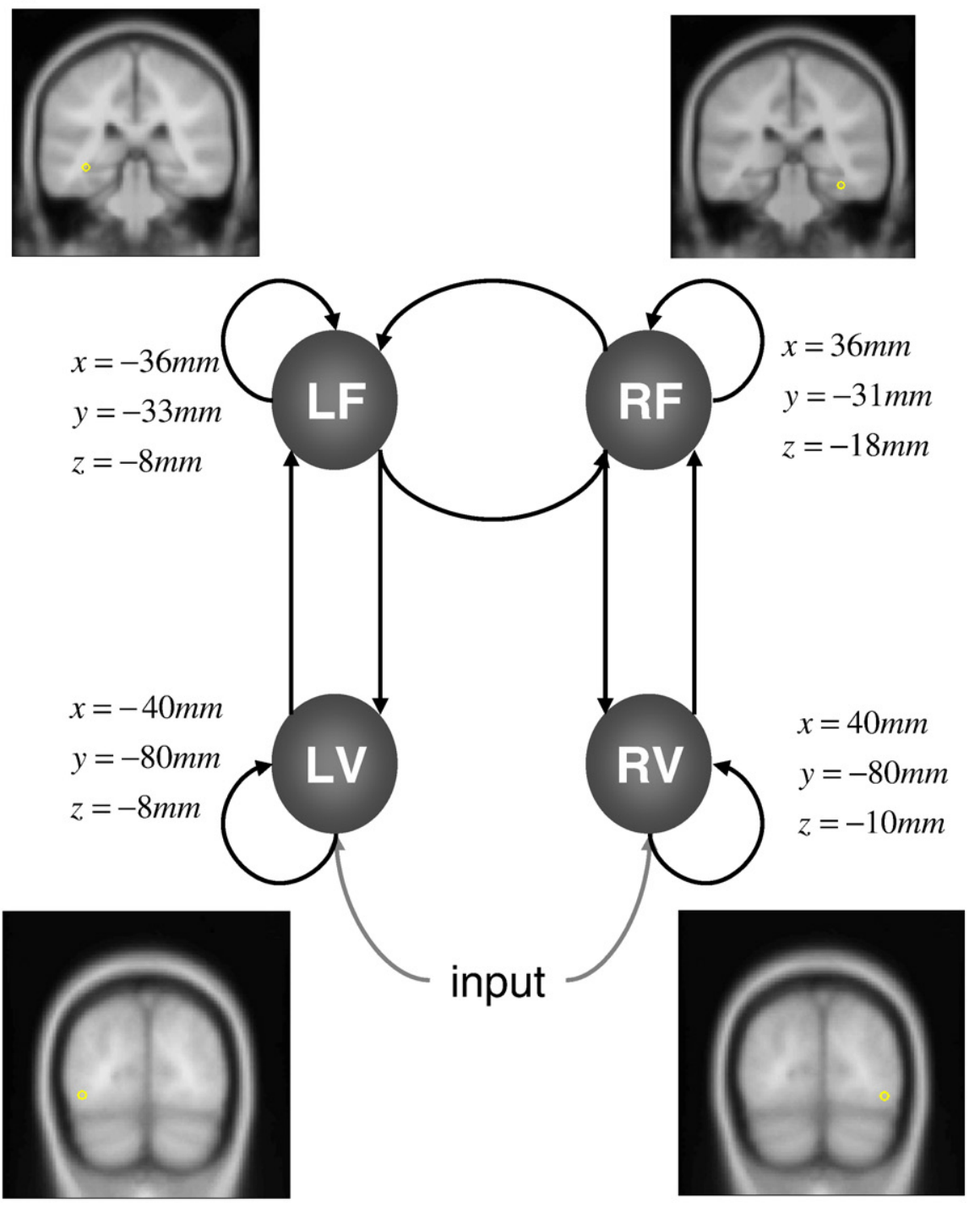

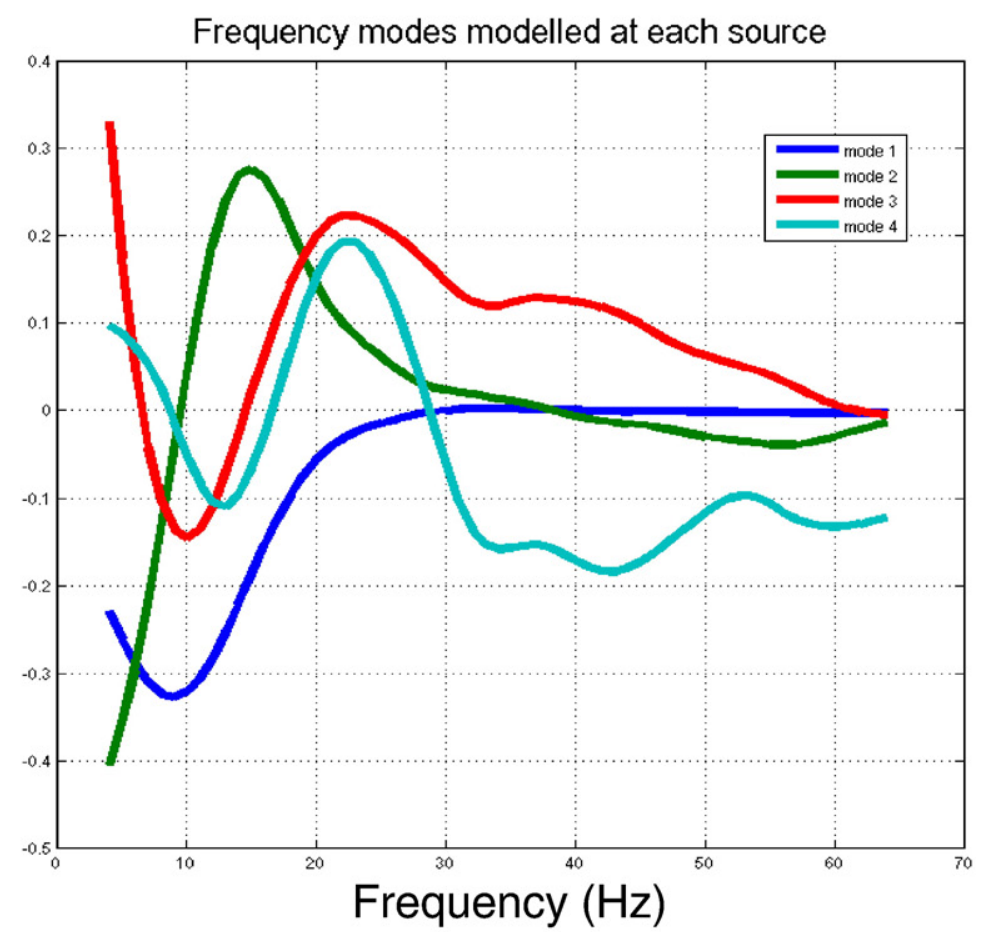

Fig. 7. Real EEG data analysis. Left (a): System or graph underlying the DCM (RV — right visual; $\mathbf{R F}$ - right fusiform; $\mathbf{L V}$ — left visual; $\mathbf{R F}$ - right fusiform). Right (b): the frequency modes, $U_{i}(\omega)$, identified using singular value decomposition of spectral dynamics in source space (over time and sources). 
Table 3

Second simulations: the impact of noise level on estimation accuracy of the parameters

\begin{tabular}{|c|c|c|c|c|}
\hline \multirow[t]{2}{*}{ Non-linear DCM } & \multicolumn{4}{|c|}{ SNR (dB) } \\
\hline & 20.38 & 14.80 & 10.83 & 4.54 \\
\hline$A_{1,1}^{3,1}=1.89$ & 1.89 & 1.89 & 1.84 & 1.75 \\
\hline$A_{1,1}^{4,2}=2.05$ & 2.05 & 2.05 & 2.00 & 1.94 \\
\hline$A_{1,1}^{3,4}=-1.05$ & -1.05 & -1.05 & -1.03 & -1.19 \\
\hline$A_{2,1}^{3,1}=-1.62$ & -1.62 & -1.62 & -1.60 & -1.49 \\
\hline$A_{1,3}^{4,2}=0.10$ & 0.10 & 0.10 & 0.08 & 0.09 \\
\hline$A_{1,2}^{3,4}=0.65$ & 0.62 & 0.65 & 0.62 & 0.64 \\
\hline$A_{2,1}^{4,3}=-1.75$ & -1.75 & -1.75 & -1.76 & -1.70 \\
\hline$A_{2,1}^{2,4}=-0.97$ & -0.97 & -0.97 & $\begin{array}{l}-0.88^{*} \\
p<0.95\end{array}$ & -0.94 \\
\hline$A_{1,2}^{4,3}=0$ & 0.00 & 0.00 & 0.00 & $\begin{array}{l}0.48^{*} \\
p>0.95\end{array}$ \\
\hline $\begin{array}{l}\text { Average error for linear } \\
\text { coupling parameters }(\%)\end{array}$ & $<0.1 \%$ & $<0.1 \%$ & $2.33 \%$ & $8.60 \%$ \\
\hline $\begin{array}{l}\text { Average error for non-linear } \\
\text { coupling parameters }(\%)\end{array}$ & $<0.1 \%$ & $<0.1 \%$ & $25.3 \%$ & $20.9 \%$ \\
\hline $\begin{array}{l}\text { Average error for all } \\
\text { parameters }(\%)\end{array}$ & $<0.1 \%$ & $<0.1 \%$ & $13.8 \%$ & $14.8 \%$ \\
\hline
\end{tabular}

The first column displays the true parameters for a selected subset of linear and non-linear parameters. Each subsequent column shows their posterior mean for decreasing signal-to-noise ratios (SNR). The last three rows display the errors of the linear and non-linear parameters, averaged over connections and expressed as a percent. A typical SNR of 20 to $15 \mathrm{~dB}$ gives veridical estimates, whereas higher noise levels (i.e., SNR of 10 to 5) can lead to inappropriate inferences (as indicated by the asterisked entries, with a posterior inference that the coupling parameter was greater or less than zero).

summarized in Table 2. For non-linear data, the non-linear DCM had a greater model evidence than the linear DCM $\left(\ln B_{21}=126\right)$ and vice versa, for linear data, where the linear DCM has the higher model evidence ( $\left.\ln B_{12}=66\right)$. When data are generated under a non-linear model, the linear model simply cannot explain them, which is reflected in the relatively large difference in log-model evidences. For linear data, this difference is much smaller $\left(\ln B_{12}=66\right)$, because the non-linear fit to the data is as good as the linear one. However, the non-linear model has more parameters, which decrease the model evidence relative to the linear model, rendering it a less likely model. The agreement between the true and conditional estimates of coupling is self-evident and, under this level of noise, we would be very confident that this coupling was not zero.

\section{Model inversion under different levels of noise}

In these simulations, we use the posterior expectations computed for real EEG data set as generating model parameters. The architecture was based on the model used to analyse the data in the next section obtained during a face-presentation paradigm. This model comprised two pairs of homologous regions in the right and left hemispheres, corresponding to early visual and fusiform sources. The sources within each hemisphere were connected reciprocally, whereas only the fusiform sources were connected between hemispheres. The spectral activity in each source was expressed in four frequency modes (identified by a singular value decomposition of the real EEG data; see Fig. 7). The input enters bilaterally at the visual sources. This exogenous input introduces a burst of power that perturbs the network at a time corresponding roughly to the arrival of subcortical input conveying sensory information (about
$60 \mathrm{~ms}$ after stimulus onset). The responses were generated as described above by integrating Eq. (11) to give, for each source, dynamics in the state-space of frequency modes.

To produce observation noise at the source level we generated sensor level white noise at four different variances. We then projected these random effects to source space using the pseudo-inverse of the lead-field and finally transformed it into the time-frequency domain (c.f., Eq. (16)). After projection onto the frequency modes we added the resulting noise spectra to the simulated source spectra. We quantified the resulting noise levels, in source space, in terms of signal-to-noise ratio (SNR) at 20.38, 14.8, 10.83, and $4.54 \mathrm{~dB}$; where we take $14.8 \mathrm{~dB}$ as representative of typical data. For example, our real EEG data had an estimated SNR of $19.74 \mathrm{~dB}$. Table 3 summarizes

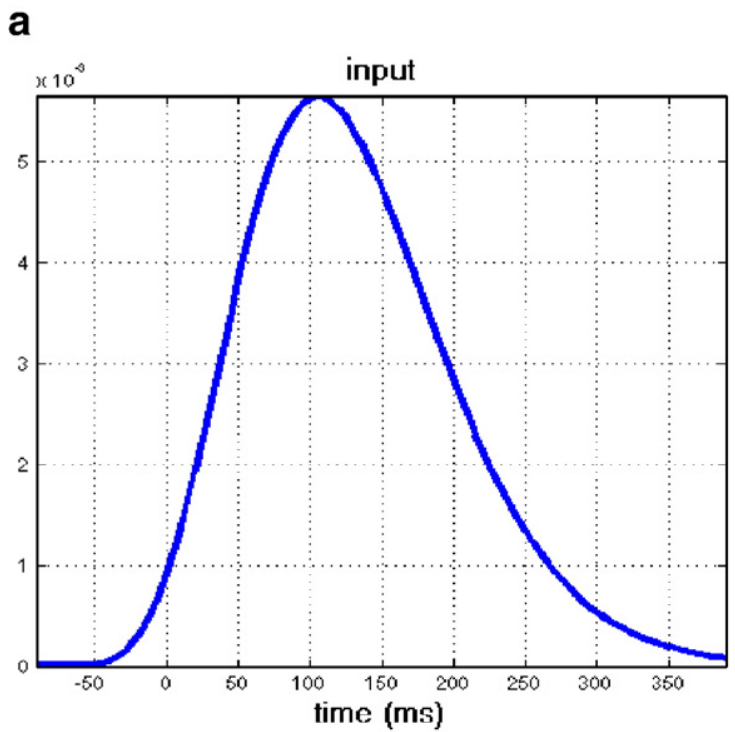

\section{Estimated inputs over peristimulus time}

b

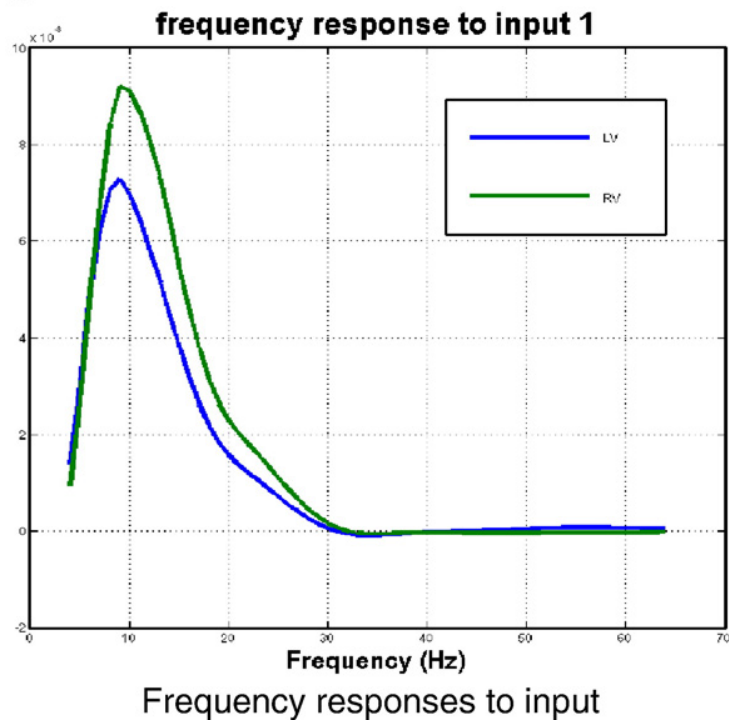

Fig. 8. Inputs to the DCM of real EEG data: (a): estimated time course of inputs to $\mathbf{R V}$ and $\mathbf{L V}$ based on the conditional means of the input function (Eq. (18)). (b). Spectral response to input in the same areas. These profiles correspond to $U C_{i}$. 


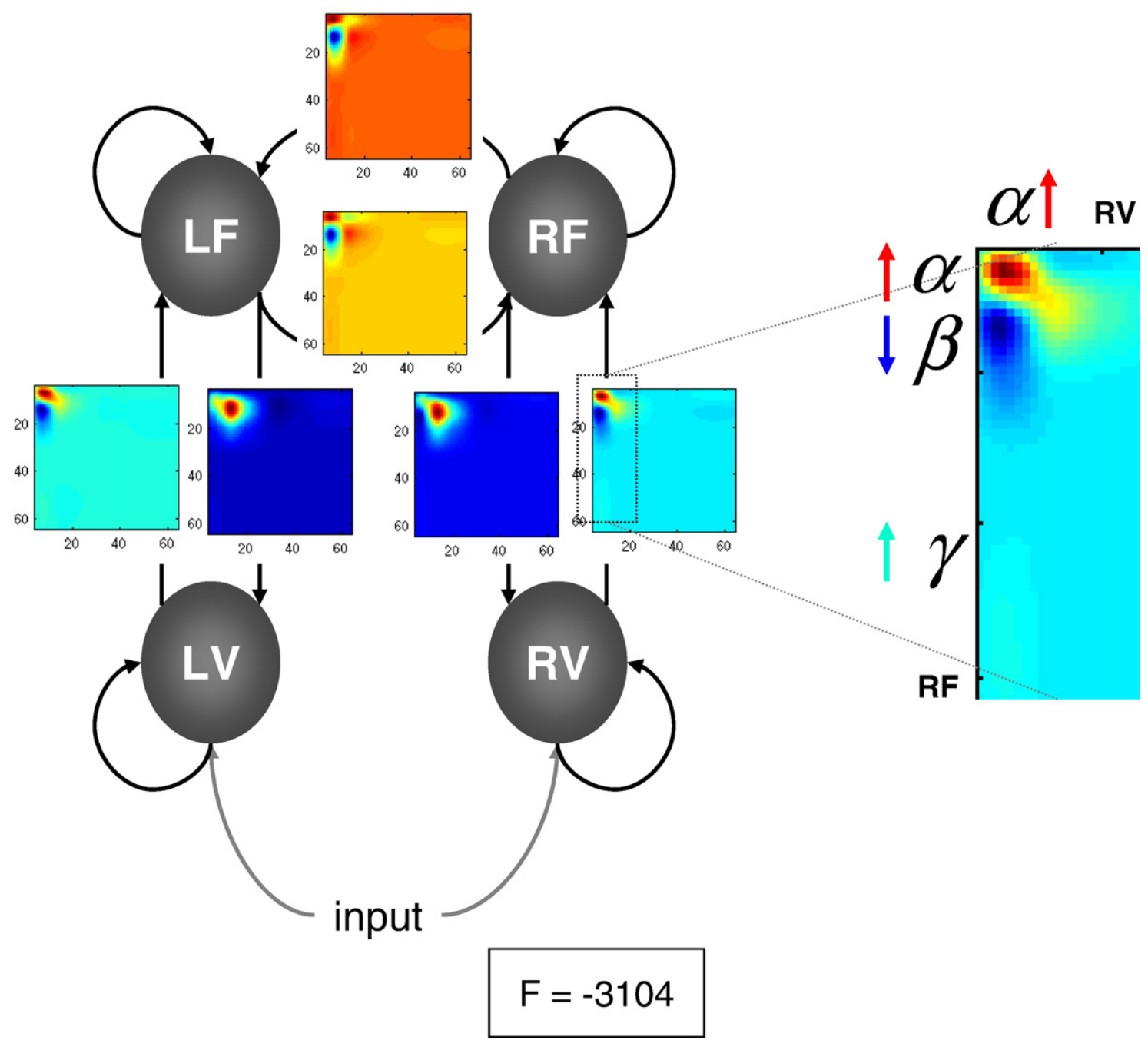

Fig. 9. Results for non-linear DCM of real EEG data: The arrows show the directed connections from one source to another. The coupling strengths are represented as coupling functions of frequency, which show the effects the spectral density in one source has on the density in another. The source names are as in Fig. 7.

the results of model inversion in terms of (i) selected posterior expectations and (ii) average errors on the linear and non-linear coupling parameters (i.e., differences between the true values and conditional expectations). These results suggest that, when the SNR is greater than $15 \mathrm{~dB}$, the connection strengths are estimated with a high degree of accuracy. As anticipated, the errors increase with noise. When the SNR is too low, inference can change in a qualitative sense. For example, at $4.54 \mathrm{~dB}$, we found that one non-linear coupling was a posteriori very likely to be present $(p>0.95)$, although the true parameter was zero.

\section{Analyses of real EEG data}

In this section, we demonstrate DCM for induced responses using EEG data. The data represents a single-subject data set from a study by Henson et al. (2003). ${ }^{6}$ The subject performed a judgement task on faces and scrambled faces. The data were sampled at $200 \mathrm{~Hz}$ using a whole-head, 128-channel ActiveTwo system. Bipolar hori-

\footnotetext{
${ }^{6}$ These data are available from http://www.fil.ion.ucl.ac.uk/spm/data/ mmfaces.html.
}

zontal and vertical electro-oculograms (EOG) were obtained using electrodes placed at the bilateral outer canthi and the left eye respectively to exclude trials with an EOG artefact. The data used here comprise time-frequency responses averaged over 86 trials.

The lead-field or gain matrix was computed for a canonical mesh (Mattout et al., 2007) and co-registered channel locations, using a three-sphere head model as encoded in BrainStorm (http://neuroimage. usc.edu/brainstorm/). The co-registration and forward model was computed within SPM5 (http://www.fil.ion.ucl.ac.uk/spm).

\section{Exemplar analysis using DCM}

Note that this single-subject analysis is used only to illustrate DCM for induced responses; we will not attempt a neurobiological interpretation of our results. Furthermore, face perception is not necessarily the most interesting paradigm, in terms of induced responses. We used these data because they are easily available (from http://www.fil.ion.ucl.ac.uk/spm), which means other people can reproduce the analysis reported below.

The specification of a DCM, i.e., the network and source locations, is a critical step. We envisage that, for a given study, there would be 
several competing models that one might want to test. In the current framework, one does this by specifying models in terms of their connections and whether these connections are linear or non-linear. Model comparison ${ }^{7}$ can then be used to select the best model and inferences about the parameters of the selected model can proceed using the conditional mean and covariance of the coupling parameters. Here, we will simply test two models to illustrate model specification, comparison, and inference. We used prior knowledge about sources in visual and fusiform cortices and employed source reconstruction implemented in SPM5 to localise four sources from a conventional ERP analysis of the data (Friston et al., 2008). These source comprised the left and right visual cortex ( $\mathbf{L V}$ and $\mathbf{R V}$ ), and left and right fusiform area (LF and $\mathbf{R F}$ ). The locations of these sources are provided in Fig. 7a, in canonical space. For these sources, spectral changes, in several frequency bands, have been found during face processing (Klopp et al., 1999). Spectra were constructed from $-100 \mathrm{~ms}$ to $400 \mathrm{~ms}$. We used a Morlet wavelet transform with a coefficient, $k=7$, over 4 to $64 \mathrm{~Hz}$. The resulting spectra were detrended and reduced using four principal modes as described above (see Fig. 7b). In the first (non-linear) DCM, we allowed bi-directional cross-frequency coupling between source pairs $\mathbf{L F}-\mathbf{R F}, \mathbf{L V}-\mathbf{L F}$, and $\mathbf{R V}-\mathbf{R F}$. In the second (linear) model, we allowed only withinfrequency interactions among these sources. We use two exogenous inputs to $\mathbf{L V}$ and $\mathbf{R V}$. The (estimated) temporal dynamics and spectral effects of these inputs on both visual sources are shown in Fig. 8.

\section{Results}

Fig. 9 summarizes the results based on the non-linear DCM. The arrows indicate directed connections. Coupling strengths are represented as functions of source and target frequency (c.f., Fig. 6). We only show coupling matrices for which one or more of the underlying coupling parameters was greater than zero, with $95 \%$ confidence or more. These matrices encode the coupling among frequencies; for example, there are several cross-frequency influences in the forward connection from $\mathbf{R V}$ to $\mathbf{R F}$, in which alpha $(8-12 \mathrm{~Hz}$ ) in the fusiform source is induced by alpha in the visual source. However, the same alpha suppresses beta $(16 \mathrm{~Hz})$, while increasing fusiform gamma power. These changes recapitulate the simulations in Fig. 4, where low frequencies in the input produce high frequency responses.

Fig. 10 shows the equivalent results for the linear DCM. In this case, only the forward connections and one transcallosal connection contained parameters that were greater then zero (at 95\% confidence). This model is largely constrained to predicting the dynamics of alpha power and is unable to account for any cross-frequency effects.

Bayesian model comparison clearly favoured the non-linear model with a log-Bayes factor of 392. Fig. 11 shows the observed and predicted spectral densities of the selected (non-linear) model. Using these spectral densities, we estimated the SNR to be $19.74 \mathrm{~dB}$. Interestingly, the coupling strengths in the right hemisphere were stronger than those in the left. Previous studies have found a right lateralisation for face processing (Kanwisher and Yovel, 2006). One can directly test this with DCM, using contrasts; for example, we found that the difference between the left and right (right minus left), averaged over all cross-frequency coupling parameters, is greater than zero, with a posterior confidence of $99 \%$. In summary, using DCM

\footnotetext{
${ }^{7}$ Note that due to the feature selection (Eq. (16)) one cannot compare models based on different lead-fields. In other words, models can only be compared if they include the same sources.
}

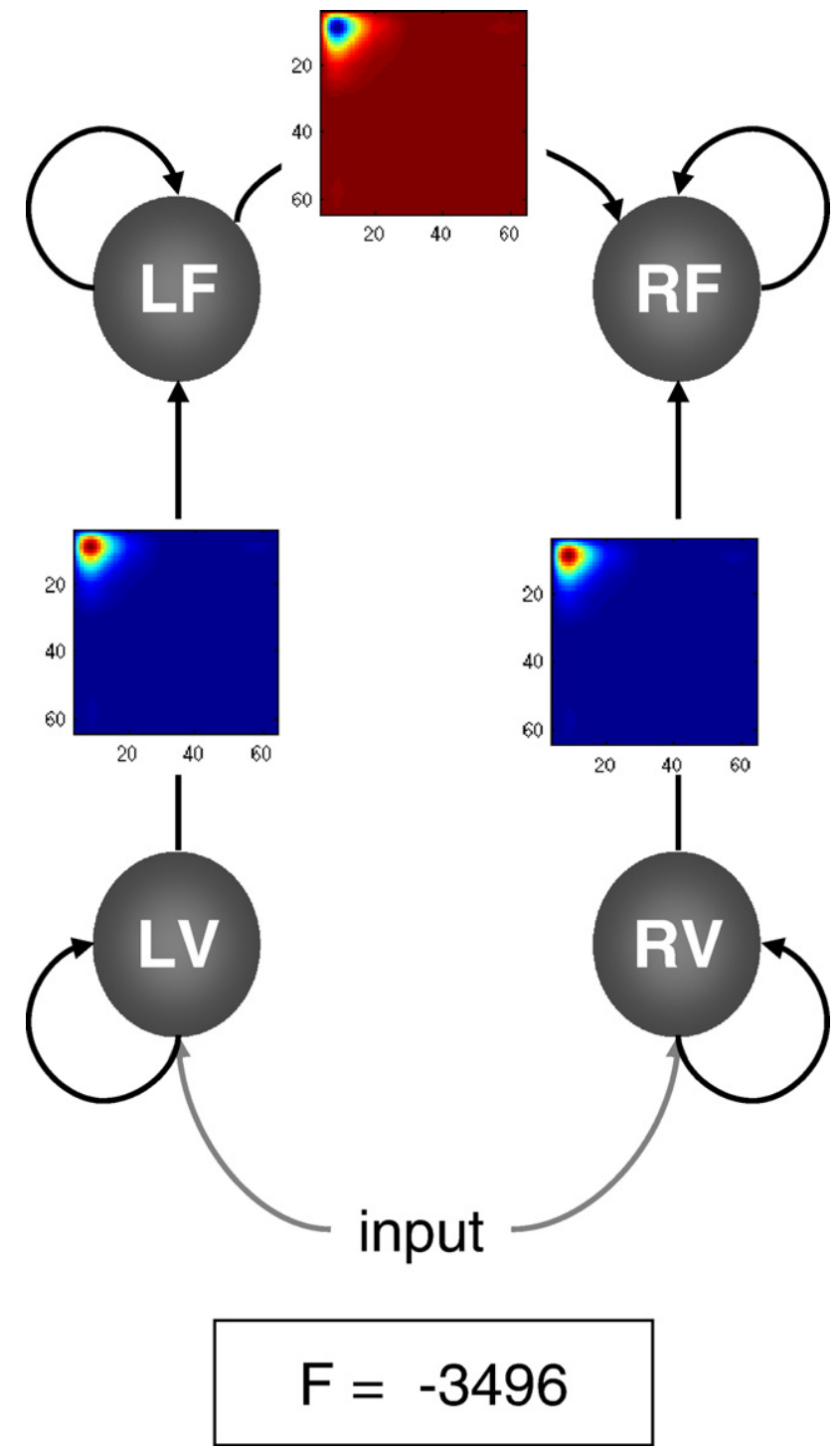

Fig. 10. Results for linear DCM of real EEG data: As for Fig. 8 but for a reduced linear model. The source names are as in Fig. 7.

and model comparison we find strong evidence for right-lateralised non-linear coupling among early visual and fusiform sources.

\section{Discussion}

Non-linearities in neuronal activity are an important aspect of processing in large-scale neuronal networks and have led to many different proposals of how to best characterise them given some data (e.g., David et al., 2004). As we have illustrated above, linear coupling is mediated by first-order transfer functions that transfer energy in the source to the target, while non-linear mechanisms express themselves as cross-frequency interactions, through highorder, generalised transfer functions. A simple example of this is frequency doubling when one squares a sinusoid; i.e., $\exp (j \omega t)^{2}=$ $\exp (j 2 \omega t)$ (see Friston (2001)). Biological evidence speaks to the prevalence of non-linear interactions among cortical areas during cognitive tasks (e.g., Bullock et al., 1997; Schack et al., 2002). We have shown that second-order features of the data (i.e., the spectrum) can be modelled by DCM for induced responses in a 

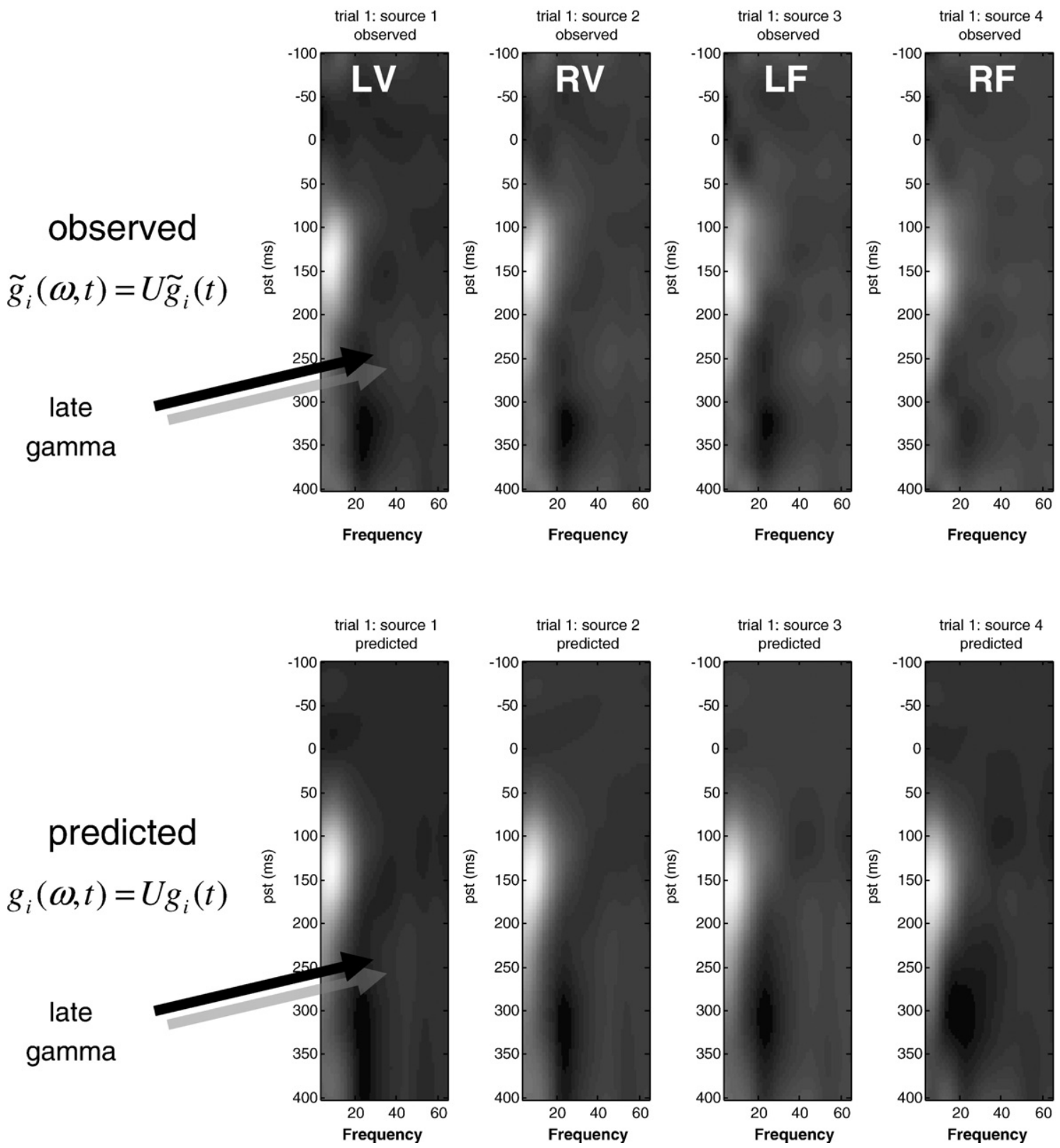

Fig. 11. Upper panels: Observed EEG time-frequency power data for all four sources. Lower panels: Fitted data. It can be seen that the model captures the main spectral dynamics fairly well. There is pronounced alpha activity around $140 \mathrm{~ms}$ with a partial return to pre-stimulus levels by $260 \mathrm{~ms}$. This corresponds largely to the evoked components. Although not very easy to see, there is also a late increase in gamma power that starts around $250 \mathrm{~ms}$ (arrow). The images correspond to the observed and predicted quantities $U \tilde{g}_{i}(t)$ and $U g_{i}(t)$ respectively.

way that can disambiguate between linear and non-linear coupling. DCM is not for a surrogate for widely used linear models (e.g., coherence, correlations) but represents a complementary approach to disclose cross-frequency interactions among areas (see also Pereda et al. (2005)).

In this paper, we have assumed that the locations of the sources are known. This means there is no source-reconstruction problem and no spatial parameters to optimise. The specification of source locations is itself a large area both in terms of evoked (Baillet and Garnero, 1997) and induced responses (Singh et al., 2003). In this work, our source locations were based on previous analyses of the data used using multiple sparse priors on distributed forward models (Friston et al., 2008). We would advocate that whatever source-reconstruction technique is used, only the locations should be retained and used to re-estimate source activity using the forward model provided by ensuing the ECD forward model. This is because the assumptions implicit in distributed forward models can introduce (e.g., though smoothness constraints) or remove (e.g., through beam-forming assumptions; Singh et al., 2003) correlations among neighbouring sources. Inverting a simple ECD forward model also ensures the DCM is insensitive to the reconstruction scheme used to define the ECD locations.

Our model furnishes a framework within which one can make inferences about causal coupling. Note that model-free approaches cannot be used to make causal inferences in a control theory sense. For example, coherence and mutual information measure inter- 


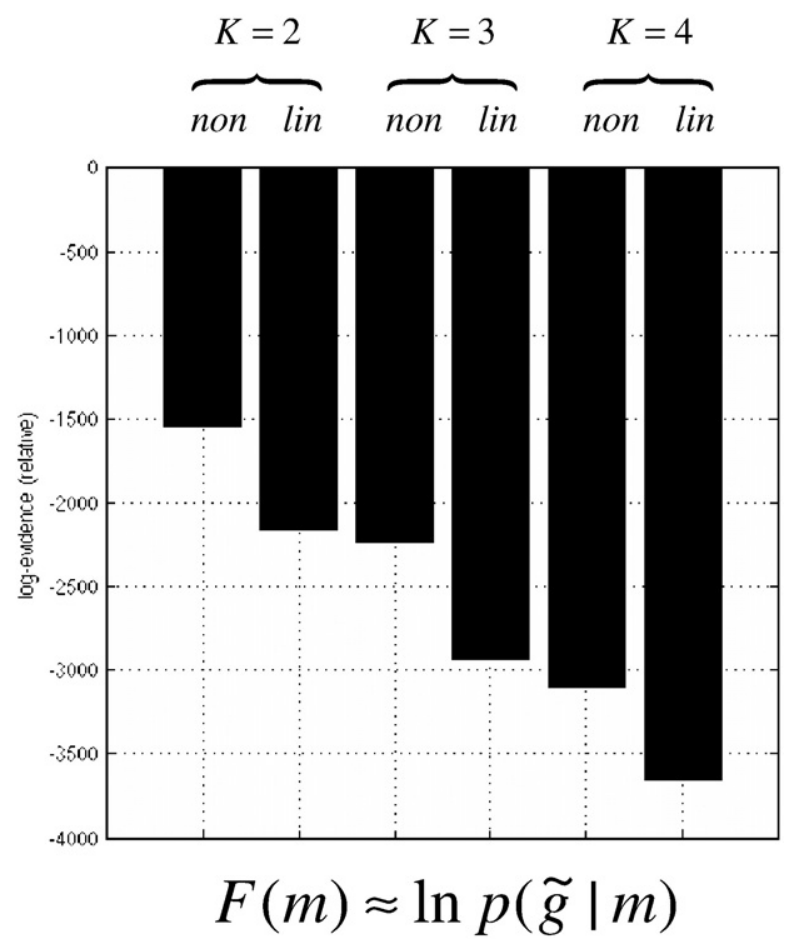

Fig. 12. Model comparison: The effect of using a different number of modes on the log-evidence for the linear and non-linear DCM. Importantly, the relative log-evidence remain stable when we change the number of modes.

dependency between time-series obtained from two sources but provide no information about directionality. Despite their names, extensions of these methods, such as Granger causality and transfer entropy, do not provide evidence for causality in a formal sense because they are based on multivariate autoregressive models, which may be causal or acausal. However, an advantage of modelfree approaches ${ }^{8}$ is that they can usually be applied in an exploratory fashion. Hypotheses about coupling, generated by these analyses, can then be tested formally using a DCM.

An important feature of DCM for induced responses is that it models the full time-frequency spectrum. This differs from typical approaches, which select a priori a few specific frequency bands. We model spectral dynamics in terms of a mixture of frequency modes (obtained with singular value decomposition). The dynamics of each mode are encoded by the evolution of a state. It is this multi-state vector, for each source, that captures how the energy in different frequencies interacts, either linearly or non-linearly, among sources. A critical issue is whether inferences differ with the number of frequency modes per source. In Fig. 12, we show that the exact number does not seem to have an effect on inference. Using a synthetic dataset (the second set of simulations with an SNR of 15), generated with three modes, we find that the non-linear DCM is always the best model for different numbers of modes. In principle, choosing too many modes should not affect inference (as shown above; Fig. 12), because parameters that relate to superfluous states will not explain data but only decrease the evidence of the model. In practice, we suggest people use as many modes as necessary to

\footnotetext{
${ }^{8}$ By model-free we mean any technique based simply on probability distributions; noting that these distributions may be parameterised with a model (e.g., autoregressive models).
}

represent $99 \%$ of the data variance. The obvious minimum to model interesting dynamics is two modes. Note that one cannot compare models with different number of modes (because the log-evidence is a function of the data features, which are defined in terms of modes).

Oscillatory activity is a widespread feature of many neuronal systems. Fluctuations in power and phase-synchronisation have been shown to be essential aspects of network function. It is widely believed that changes in the synchronous discharge of neuronal assemblies contribute to the increase or decrease of power within taskrelated frequencies (Kilner et al., 2003; Pfurtscheller and Lopes da Silva, 1999). Conversely, methods for analysing phase-synchrony assess the stability of the phase-difference within a certain period (e.g., Lachaux et al., 1999; Rosenblum et al., 1996). High phasesynchronisation is thought to indicate transient functional coupling among cortical areas (Kopell et al., 2000); c.f., the "binding problem" (Gray et al., 1989; Varela et al., 2001). Although phase and power appear to be two different phenomena, they must of course be related by some common generative model. This paper presents the first step towards generative models of changes in power and the nature of the implicit coupling. Over the next year, we hope to complement DCM for induced power with a DCM for phase-coupling. The overall approach will be to recapitulate the developments in this paper but replacing the spectral power with instantaneous phase (using the phase of the integral in Eq. (9), as opposed to its absolute value). We can then use non-linear [periodic] functions of the phase-difference between two sources (c.f., Eq. (1)) as the basis of a likelihood model. By modelling electromagnetic sources as an ensemble of oscillators with different intrinsic frequencies, we can ask question about phaseentrainment, nested oscillations and other key phenomena that have been used to study non-linear coupling in the brain.

\section{Conclusion}

Non-linear interactions are an important phenomenon in the brain and are expressed as cross-frequency coupling in spectral characterisations of EEG and MEG time-series. Dynamic causal modelling for induced responses exploits this to model dynamic broad-band power changes as a consequence of linear and non-linear coupling among brain sources. The ensuing scheme might be useful when trying to disambiguate linear and non-linear contributions to distributed processing in a network of electromagnetic sources.

\section{Software note}

The scheme described in this note has been implemented as Matlab (MathWorks) code. The source code is available as a toolbox ('api_erp') in the Statistical Parametric Mapping package (SPM5) from http://www.fil.ion.ucl.ac.uk/spm/ as academic freeware.

\section{Acknowledgments}

The Wellcome Trust and National Science Council of Taiwan (TMS-094-1-A-037) funded this work. We would also like to thank Dr. James Kilner for his valuable support, and Marcia Bennett for helping prepare this manuscript.

\section{References}

Baillet, S., Garnero, L.A., 1997. Bayesian approach to introducing anatomofunctional priors in the EEG/MEG inverse problem. IEEE Trans. Biomed. Eng. 44, 374-385. 
Bendat, J.S., 1990. Nonlinear System Analysis and Identification from Random Data. John Wiley and Sons, New York USA.

Bernasconi, C., Konig, P., 1999. On the directionality of cortical interactions studied by structural analysis of electrophysiological recordings. Biol. Cybern. 81, 199-210.

Bullock, T.H., Achimowicz, J.Z., Duckrow, R.B., Spencer, S.S., Iragui-Madoz, V.J., 1997. Bicoherence of intracranial EEG in sleep, wakefulness and seizures. Electroencephalogr. Clin. Neurophysiol. 103, 661-678.

Breakspear, M., Terry, J.R., 2002. Detection and description of non-linear interdependence in normal multichannel human EEG data. Clin. Neurophysiol. 113, 735-753.

Bressler, S., 1995. Large-scale cortical networks and cognition. Brain Res. Rev. 20, 288-304.

Brovelli, A., Ding, M., Ledberg, A., Chen, Y., Nakamura, R., Bressler, S.L., 2004. Beta oscillations in a large-scale sensorimotor cortical network: directional influences revealed by Granger causality. Proc. Natl. Acad. Sci. U. S. A. 101, 9849-9854.

Czigler, B., Csikos, D., Hidasi, Z., Anna Gaal, Z., Csibri, E., Kiss, E., 2008. Quantitative EEG in early Alzheimer's disease patients - power spectrum and complexity features. Int. J. Psychophysiol. 68, 75-80.

Darvas, F., Pantazis, D., Kucukaltun-Yildirim, E., Leahy, R.M., 2004. Mapping human brain function with MEG and EEG: methods and validation. NeuroImage 23 (Suppl 1), S289-S299.

David, O., Cosmelli, D., Lachaux, J.-P., Baillet, S., Garnero, L., Martinerie, J., 2003. A theoretical and experimental introduction to the non-invasive study of large-scale neural phase synchronization in human beings. Int. J. Comput. Cogn. 1, 53-77.

David, O., Cosmelli, D., Friston, K.J., 2004. Evaluation of different measures of functional connectivity using a neural mass model. NeuroImage 21 (2), 659-673 (Feb).

David, O., Kiebel, S., Harrison, L.M., Mattout, J., Kilner, J.M., Friston, K.J., 2006a. Dynamic causal modelling of evoked responses in EEG and MEG. NeuroImage 20, 1255-1272 pp.

David, O., Kilner, J.M., Friston, K.J., 2006b. Mechanisms of evoked and induced responses in MEG/EEG. NeuroImage 31, 1580-1591.

Dumermuth, G., Huber, P.J., Kleiner, B., Gasser, T., 1971. Analysis of interrelations between frequency bands of EEG by means of bispectrum preliminary study. Electroencephalogr. Clin. Neurophysiol. 31, 137-148.

Ermentrout, G.B., Kleinfeld, D., 2001. Traveling electrical waves in cortex insights from phase dynamics and speculation on a computational role. Neuron 29, 33-44 (Vol.).

Engel, A.K., Fries, P., Singer, W., 2001. Dynamic predictions: oscillations and synchrony in top-down processing. Nat. Rev., Neurosci. 2, 704-716.

Fliess, M., Lamnabhi, M., Lamnabhi-Lagarrigue, F., 1983. An algebraic approach to nonlinear functional expansions. IEEE Trans. Circuits Syst. $30,554-570$.

Friston, K.J., 1997. Another neural code? NeuroImage 5, 213-220.

Friston, K.J., 2000. The labile brain I: neuronal transients and nonlinear coupling. Philos. Trans. R. Soc. Lond. 355, 215-236.

Friston, K.J., 2001. Brain function, nonlinear coupling, and neuronal transients. Neuroscientist 7, 406-418.

Friston, K.J., Harrison, L., Penny, W., 2003. Dynamic causal modelling. NeuroImage 19, 1273-1302.

Friston, K., Henson, R., Phillips, C., Mattout, J., 2006. Bayesian estimation of evoked and induced responses. Hum. Brain Mapp. 27, 722-735.

Friston, K., Harrison, L., Daunizeau, J., Kiebel, S., Phillips, C., TrujilloBarreto, N., Henson, R., Flandin, G., Mattout, J., 2008. Multiple sparse priors for the M/EEG inverse problem. NeuroImage 39, 1104-1120.

Gerloff, C., Andres, F.G., 2002. Bimanual coordination and interhemispheric interaction. Acta Psychol. (Amst.) 110, 161-186.

Gray, C.M., Konig, P., Engel, A.K., Singer, W., 1989. Oscillatory responses in cat visual cortex exhibit inter-columnar synchronization which reflects global stimulus properties. Nature 338, 334-337.

Gross, J., Kujala, J., Hamalainen, M., Timmermann, L., Schnitzler, A., Salmelin, R., 2001. Dynamic imaging of coherent sources: studying neural interactions in the human brain. Proc. Natl. Acad. Sci. U. S. A. 98, 694-699.
Guderian, S., Duzel, E., 2005. Induced theta oscillations mediate large-scale synchrony with mediotemporal areas during recollection in humans. Hippocampus 15, 901-912.

Henson, R.N., Goshen-Gottstein, Y., Ganel, T., Otten, L.J., Quayle, A., Rugg, M.D., 2003. Electrophysiological and haemodynamic correlates of face perception, recognition and priming. Cereb. Cortex 13, 793-805.

Hirsch, J.A., Martinez, L.M., Alonso, J.M., Desai, K., Pillai, C., Pierre, C., 2002. Synaptic physiology of the flow of information in the cat's visual cortex in vivo. J. Physiol. 540 (Pt 1), 335-350 (Apr 1).

Jansen, B.H., Rit, V.G., 1995. Electroencephalogram and visual evoked potential generation in a mathematical model of coupled cortical columns. Biol. Cybern. 73, 357-366.

Jeffrey, C.S., Chamoun, N.G., 1994. An introduction to bispectral analysis for the electroencephalogram. J. Clin. Monit. 10, 392-404.

Kanwisher, N., Yovel, G., 2006. The fusiform face area: a cortical region specialized for the perception of faces. Philos. Trans. R. Soc. Lond., B Biol. Sci. 361, 2109-2128.

Kiebel, S.J., David, O., Friston, K.J., 2006. Dynamic causal modelling of evoked responses in EEG/MEG with lead field parameterization. NeuroImage 30 (4), 1273-1284 (May).

Kiebel, S.J., Daunizeau, J., Phillips, C., Friston, K.J., 2008. Variational Bayesian inversion of the equivalent current dipole model in EEG/MEG. NeuroImage 39, 728-741.

Kilner, J.M., Alonso-Alonso, M., Fisher, R., Lemon, R.N., 2002. Modulation of synchrony between single motor units during precision grip tasks in humans. J. Physiol. 541, 937-948.

Kilner, J.M., Salenius, S., Baker, S.N., Jackson, A., Hari, R., Lemon, R.N., 2003. Task-dependent modulations of cortical oscillatory activity in human subjects during a bimanual precision grip task. NeuroImage 18, 67-73.

Kilner, J.M., Paulignan, Y., Boussaoud, D., 2004. Functional connectivity during real vs imagined visuomotor tasks: an EEG study. NeuroReport $15,637-642$.

Kilner, J.M., Mattout, J., Henson, R., Friston, K.J., 2005. Hemodynamic correlates of EEG: a heuristic. NeuroImage 28, 280-286.

Klopp, J., Halgren, E., Marinkovic, K., Nenov, V., 1999. Face-selective spectral changes in the human fusiform gyrus. Clin. Neurophysiol. 110, 676-682.

Kopell, N., Ermentrout, G.B., 1986. Symmetry and phaselocking in chains of weakly coupled oscillators. Commun. Pure Appl. Math. 39, 623-660.

Kopell, N., Ermentrout, G.B., Whittington, M.A., Traub, R.D., 2000. Gamma rhythms and beta rhythms have different synchronization properties. Proc. Natl. Acad. Sci. U. S. A. 97, 1867-1872.

Kuramoto, Y., 1984. Chemical Oscillations, Waves and Turbulence. Springer Verlag, New York.

Lachaux, J.P., Rodriguez, E., Martinerie, J., Varela, F.J., 1999. Measuring phase synchrony in brain signals. Hum. Brain Mapp. 8, 194-208.

Lee, K.H., Williams, L.M., Breakspear, M., Gordon, E., 2003. Synchronous gamma activity: a review and contribution to an integrative neuroscience model of schizophrenia. Brain Res. Brain Res. Rev. 41, 57-78.

Leocani, L., Toro, C., Manganotti, P., Zhuang, P., Hallett, M., 1997. Event-related coherence and event-related desynchronization/synchronization in the $10 \mathrm{~Hz}$ and $20 \mathrm{~Hz}$ EEG during self-paced movements. Electroencephalogr. Clin. Neurophysiol. 104, 199-206.

Mattout, J., Phillips, C., Penny, W.D., Rugg, M.D., Friston, K.J., 2006. MEG source localization under multiple constraints: an extended Bayesian framework. NeuroImage 30, 753-767.

Mattout, J., Henson, R.N., Friston, K.J., 2007. Canonical source reconstruction for MEG. Comput. Intell. Neurosci. 1, 17-27.

Michel, C.M., Murray, M.M., Lantz, G., Gonzalez, S., Spinelli, L., Grave de Peralta, R., 2004. EEG source imaging. Clin. Neurophysiol. 115, 2195-2222.

Moran, R.J., Kiebel, S.J., Stephan, K.E., Reilly, R.B., Daunizeau, J., Friston, K.J., 2007. A neural mass model of spectral responses in electrophysiology. NeuroImage 37, 706-720.

Nunez, P.L., Srinivasan, R., Westdorp, A.F., Wijesinghe, R.S., Tucker, D.M., Silberstein, R.B., Cadusch, P.J., 1997. EEG coherency. I: statistics, reference electrode, volume conduction, Laplacians, cortical imaging, 
and interpretation at multiple scales. Electroencephalogr. Clin. Neurophysiol. 103, 499-515.

Palva, J.M., Palva, S., Kaila, K., 2005. Phase synchrony among neuronal oscillations in the human cortex. J. Neurosci. 25, 3962-3972.

Pantev, C., 1995. Evoked and induced gamma-band activity of the human cortex. Brain Topogr. 7, 321-330.

Patino, L., Chakarov, V., Schulte-Monting, J., Hepp-Reymond, M.C., Kristeva, R., 2006. Oscillatory cortical activity during a motor task in a deafferented patient. Neurosci. Lett. 401, 214-218.

Penny, W.D., Stephan, K.E., Mechelli, A., Friston, K.J., 2004. Comparing dynamic causal models. NeuroImage 22, 1157-1172.

Pereda, E., Quiroga, R.Q., Bhattacharya, J., 2005. Nonlinear multivariate analysis of neurophysiological signals. Prog. Neurobiol. 77, 1-37.

Pfurtscheller, G., Lopes da Silva, F.H., 1999. Event-related EEG/MEG synchronization and desynchronization: basic principles. Clin. Neurophysiol. 110, 1842-1857.

Pikovsky, A., Rosenblum, M., Kurths, J., 2001. Synchronization. A Universal Concept in Nonlinear Sciences. Cambridge University Press, Cambridge.

Priestley, M.B., 1988. Non-linear and Non-stationary Time Series Analysis. Academic Press, London.

Quian Quiroga, R., Kraskov, A., Kreuz, T., Grassberger, P., 2002. Performance of different synchronization measures in real data: a case study on electroencephalographic signals. Phys. Rev., E 65, 041903.

Raethjen, J., Govindan, R.B., Kopper, F., Muthuraman, M., Deuschl, G., 2007. Cortical involvement in the generation of essential tremor. J. Neurophysiol. 97, 3219-3228.

Rosenblum, M., Pikovsky, A., Kurths, J., 1996. Phase synchronization of chaotic oscillators. Phys. Rev. Lett. 76, 1804-1807.

Roulston, M.S., 1999. Estimating the errors on measured entropy and mutual information. Physica, D 125, 285-294.

Rowe, D.L., Robinson, P.A., Gordon, E., 2005. Stimulant drug action in attention deficit hyperactivity disorder (ADHD): inference of neurophysiological mechanisms via quantitative modelling. Clin. Neurophysiol. 116 (2), 324-335 (Feb).

Rulkov, N.F., Sushchik, M.M., Tsimring, L.S., Abarbanel, H.D., 1995. Generalized synchronization of chaos in directionally coupled chaotic systems. Phys. Rev., E 51, 980-994.
Schack, B., Vath, N., Petsche, H., Geissler, H.G., Moller, E., 2002. Phasecoupling of theta-gamma EEG rhythms during short-term memory processing. Int. J. Psychophysiol. 44, 143-163.

Schiff, S.J., So, P., Chang, T., Burke, R.E., Sauer, T., 1996. Detecting dynamical interdependence and generalized synchrony through mutual prediction in a neural ensemble. Phys. Rev., E 54, 6708-6724.

Shils, J.L., Litt, M., Skolnick, B.E., Stecker, M.M., 1996. Bispectral analysis of visual interactions in humans. Electroencephalogr. Clin. Neurophysiol. 98, $113-125$

Singer, W., 1999. Neuronal synchrony: a versatile code for the definition of relations? Neuron 24, 49-65.

Singh, K.D., Barnes, G.R., Hillebrand, A., Forde, E.M., Williams, A.L., 2002. Task-related changes in cortical synchronization are spatially coincident with the hemodynamic response. NeuroImage 16 (1), 103-114.

Singh, K.D., Barnes, G.R., Hillebrand, A., 2003. Group imaging of taskrelated changes in cortical synchronisation using nonparametric permutation testing. NeuroImage 19, 1589-1601.

Tallon-Baudry, C., Bertrand, O., 1999. Oscillatory gamma activity in humans and its role in object representation. Trends Cogn. Sci. 3, 151-162.

Tallon-Baudry, C., Bertrand, O., Delpuech, C., Permier, J., 1997. Oscillatory gamma-band $(30-70 \mathrm{~Hz})$ activity induced by a visual search task in humans. J. Neurosci. 17, 722-734.

Tass, P., Rosenblum, M.G., Weule, J., Kurths, J., Pikovsky, A., Volkmann, J., Schnitzler, A., Freund, H.J., 1998. Detection of $n: m$ phase locking from noisy data: application to magnetoencephalography. Phys. Rev. Lett. 81, 3291-3294.

Tiitinen, H., Sinkkonen, J., Reinikainen, K., Alho, K., Lavikainen, J., Naatanen, R., 1993. Selective attention enhances the auditory $40-\mathrm{Hz}$ transient response in humans. Nature 364, 59-60.

Vanhatalo, S., Palva, J.M., Holmes, M.D., Miller, J.W., Voipio, J., Kaila, K., 2004. Infraslow oscillations modulate excitability and interictal epileptic activity in the human cortex during sleep. Proc. Natl. Acad. Sci. U. S. A. 101, 5053-5057.

Varela, F., Lachaux, J.-P., Rodriguez, E., Martinerie, J., 2001. The brainweb: phase synchronization and large-scale integration. Nat. Rev., Neurosci. 2, 229-239.

Wright, J.J., Liley, D.T., 1994. A millimetric-scale simulation of electrocortical wave dynamics based on anatomical estimates of cortical synaptic density. Comput. Neural Syst. 5 (2), 191-202. 